\title{
The 26 January $2001 M 7.6$ Bhuj, India, Earthquake: Observed and Predicted Ground Motions
}

\author{
by Susan E. Hough, Stacey Martin, Roger Bilham, and Gail M. Atkinson
}

\begin{abstract}
Although local and regional instrumental recordings of the devastating 26, January 2001, Bhuj earthquake are sparse, the distribution of macroseismic effects can provide important constraints on the mainshock ground motions. We compiled available news accounts describing damage and other effects and interpreted them to obtain modified Mercalli intensities (MMIs) at $>200$ locations throughout the Indian subcontinent. These values are then used to map the intensity distribution throughout the subcontinent using a simple mathematical interpolation method. Although preliminary, the maps reveal several interesting features. Within the Kachchh region, the most heavily damaged villages are concentrated toward the western edge of the inferred fault, consistent with western directivity. Significant sediment-induced amplification is also suggested at a number of locations around the Gulf of Kachchh to the south of the epicenter. Away from the Kachchh region, intensities were clearly amplified significantly in areas that are along rivers, within deltas, or on coastal alluvium, such as mudflats and salt pans. In addition, we use fault-rupture parameters inferred from teleseismic data to predict shaking intensity at distances of $0-1000 \mathrm{~km}$. We then convert the predicted hard-rock ground-motion parameters to MMI by using a relationship (derived from Internet-based intensity surveys) that assigns MMI based on the average effects in a region. The predicted MMIs are typically lower by 1-3 units than those estimated from news accounts, although they do predict near-field ground motions of approximately $80 \% \mathrm{~g}$ and potentially damaging ground motions on hard-rock sites to distances of approximately $300 \mathrm{~km}$. For the most part, this discrepancy is consistent with the expected effect of sediment response, but it could also reflect other factors, such as unusually high building vulnerability in the Bhuj region and a tendency for media accounts to focus on the most dramatic damage, rather than the average effects. The discrepancy may also be partly attributable to the inadequacy of the empirical relationship between MMI and peak ground acceleration (PGA), when applied to India. The MMI-PGA relationship was developed using data from California earthquakes, which might have a systematically different stress drop and therefore, a different frequency content than intraplate events. When a relationship between response spectra and MMI is used, we obtain larger predicted MMI values, in better agreement with the observations.
\end{abstract}

\section{Introduction}

The $M$ 7.6 Bhuj earthquake occurred in the state of Gujarat, India, at 03:16 UTC (8:16 a.m., local time) on 26 January 2001 (Fig. 1). The event struck within the Kachchh peninsula near India's western coast and was felt over much of the Indian subcontinent. Official government figures placed the death toll at over 20,000 and the number of injured at 166,000. Government estimates place direct economic losses due to the earthquake at 1.3 billion dollars, although more recent estimates indicate losses as high as 5 billion.
Eyewitnesses reported that approximately one building in 10 remained standing in Bhuj and Anjar, the closest large cities to the epicenter. Considerable damage was also reported in Hyderabad in southern Pakistan, whereas cities on the ancient Indian craton at similar distances from the epicenter were not severely shaken. Although some multistory concrete buildings completely collapsed in moderately shaken regions, many other structures remained intact, indicating that poor-quality construction aggravated the damage. This was evident to provincial administrators, because 


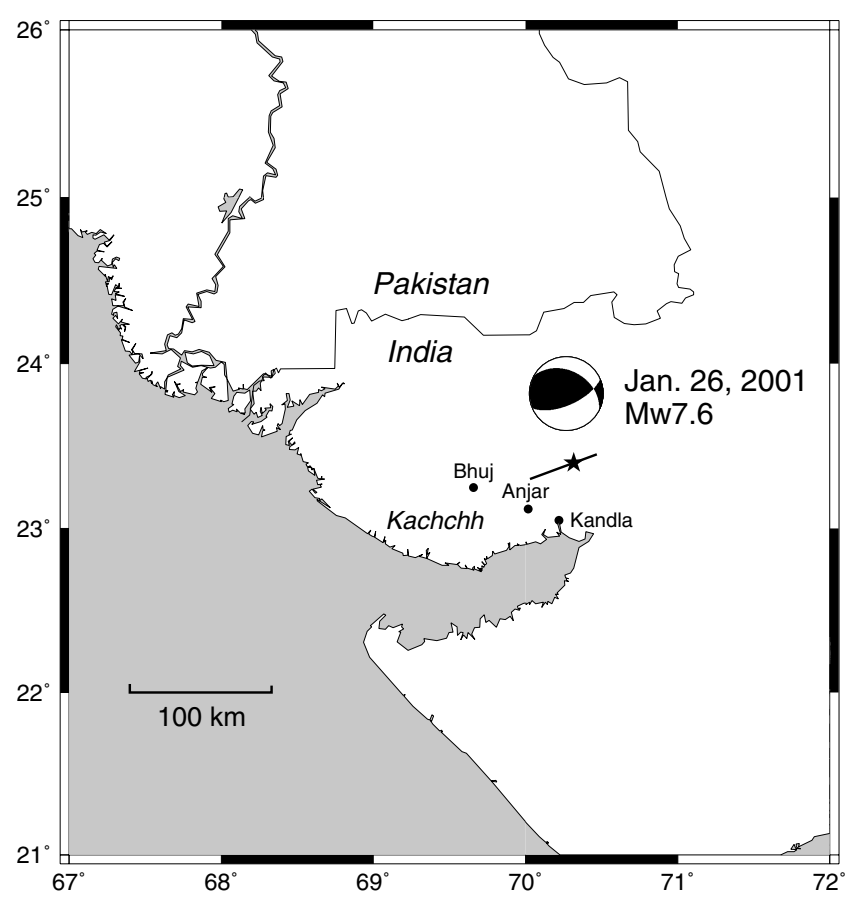

Figure 1. Map showing location of the 26 January 2001, Bhuj, India, earthquake within the Rann of Kachchh. The straight line shows a "pseudo-fault" with strike and length from Yagi and Kikuchi (2001). The focal mechanism corresponding to this solution is also shown. Preliminary aftershock relocations indicate a south-dipping rupture plane.

within a week of the event, Ahmedabad police had registered 37 cases of culpable homicide and criminal conspiracy against builders, architects, and engineers of buildings that collapsed in the earthquake.

The Bhuj earthquake generated substantial liquefaction and hydrological effects. Local hydrologists and villagers reported that the quake briefly activated desert rivers that had been dry for more than a century. Widespread liquefaction was confirmed by satellite imagery and by field observations (e.g., Tuttle et al., 2001a, b). Many mud volcanos in the Rann of Kachchh have dimensions of tens of meters; one covered a 5-km-diameter stretch of the southern Rann with dark sand and mud. Numerous ancient river channels have been illuminated by a pock-mark pattern of vented sand and water, and some have clearly flowed and breached their old channels. Roads and fields near Bhachau were ruptured by 2-3-m-wide cracks resulting from substantial lateral spreading. The Port of Kandla was severely damaged by liquefaction and related ground failures, although numerous engineered structures, such as oil tanks, survived the earthquake.

The Bhuj earthquake occurred far from the edge of the Indian plate and quite close to an $M 7.7$ earthquake that occurred in 1819 (Oldham, 1926; Bilham, 1998). The 2001 felt region extends from Madras to Kathmandu, just as it did in the 1819 earthquake. Damage reports from Bhuj and An- jar are also strikingly similar to the damage reports of the 1819 earthquake. However, $<2000$ people were killed in the 1819 event. The population of Kachchh is now many times greater than in 1819, but the percentage of the local population killed is roughly the same, despite the implementation of a seismic-resistant building code.

Although instrumental recordings of the Bhuj earthquake are unfortunately scarce, isoseismal intensities provide an important data set. The distribution of strong-motion instruments in India is not adequate to calibrate directly the modified Mercalli intensity (MMI) values relative to physical ground-motion parameters. However, the Bhuj earthquake was well recorded at teleseismic distances. Intensity results from the Bhuj earthquake will thus be useful to better constrain the magnitude of historical Indian earthquakes (e.g., Ambraseys and Bilham, 2000).

Although a full compilation of shaking effects will not be available for some time, extensive news articles were written in the early aftermath of the Bhuj earthquake and were published in both conventional newspapers and on the Web. We compiled available accounts from reputable sources and interpreted them to obtain MMI values following conventional practice. The most difficult accounts to interpret are those that describe only liquefaction and/or disruption of underground water levels. Although recent evidence has shown that such effects can occur at relatively modest shaking levels (e.g., Musson, 1998), we have assigned MMI values according to the classic definitions in part to facilitate comparisons between our values and those determined for other earthquakes. Accordingly, such sites are given MMI values of VII-VIII, although we recognize the possibility that they may not reflect the overall level of ground motion. Our final data set includes MMI values for nearly 200 sites throughout the Indian subcontinent, with the highest concentration of values within $300 \mathrm{~km}$ of Bhuj.

We anticipate that our results will eventually be supplanted by MMI maps determined from ground observations and conventional mail surveys. However, we proceed with a determination of a "media-based intensity map" for two reasons. First, we believe the map does provide a good characterization of shaking effects throughout the subcontinent. More important, however, we construct our MMI map based solely on media accounts, so that the results can be compared with both media-based maps for earlier earthquakes and with the MMI distribution determined for the Bhuj earthquake from conventional ground- and mail-based surveys. These comparisons should provide useful insights into the nature of the biases that can result from determination of intensity distribution based only on news reports. Because such sources often provide the only source of information for older earthquakes (pre-1900, typically), the issue of "media biases" often looms large in the interpretation of intensity data for important historical earthquakes. Furthermore, it is likely that Web and media-based assessments will become increasingly common in future large earthquakes worldwide. 


\section{The Bhuj Mainshock: A Brief Overview}

The preliminary focal mechanism of the Bhuj earthquake (e.g., Yagi and Kikuchi, 2001) suggests that the fault apparently occurred on a steeply dipping thrust fault that did not break the surface (Bendick et al., 2001). The estimated moment magnitude, $M_{\mathrm{w}}$, ranges from 7.5 to 7.7 , suggesting a rupture of 15-30-km width, 50-100-km length, and average slip of $1-4 \mathrm{~m}$. Preliminary results from aftershock studies indicate that the rupture was no shallower than about 8-9 km (Horton et al., 2001). The surface manifestation of such a rupture is likely to be a broad zone of distributed uplift and subsidence with secondary surface faulting and cracking.

Parallels have been noted between the Bhuj earthquake and the 1811-1812 New Madrid sequence. It remains unclear, however, if the strain rates and/or overall tectonic settings of the two regions are analagous, but both the Bhuj earthquake and the largest New Madrid event (on 7 February 1812) occurred on thrust faults that failed to produce either extensive or pronounced surface ruptures (e.g., Mueller and Pujol, 2001). According to a recent reinterpretation by Hough et al. (2000), the magnitudes of these events may also have been similar. Results from studies of the Bhuj earthquake therefore have the potential to provide important insights for earthquakes in other areas.

\section{Isoseismal Intensities}

From methods such as ground-based and mail surveys, a full compilation of shaking effects from large earthquakes is typically not available for some time after the event. In the immediate aftermath of the Bhuj earthquake, we compiled news accounts from traditional print media sources in the United States and India as well as Internet-based sources. A summary of these reports, including their sources, is listed in Table 1. From the available accounts, we assigned MMIs (e.g., Stover and Coffman, 1993) based on the severity of shaking. In a few cases, news sources document that the event was not felt at a given location. In the Kachchh region, the most heavily damaged regions are generally assigned MMI values of IX-X, corresponding to heavy damage to masonry structures. Few values in excess of $\mathrm{X}$ are assigned, reflecting the paucity of accounts describing significant damage to modern, engineered structures. In the town of Sukhpur, however, one account describes a 10-year-old child being flung into the air. We assign an MMI of XI-XII for this location.

Intensity values can be interpreted as point data; our results for the Bhuj earthquake are shown in Figure 2. Typically, however, such data are used to define isoseismal contours. This approach is fraught with potential biases, as discussed at length by Hough et al. (2000). In particular, any general approach to interpolation or contouring will not re- flect the systematic dependence of ground motions on site geology. Ideally, knowledge of local geological structure can provide important constraints, but such information is not readily available in this case.

To map the shaking distribution over the entire subcontinent, we use a simple mathematical approach, whereby the data are contoured using a continuous-curvature gridding algorithm. A uniform grid of estimated intensity values, $I(x, y)$, is determined by solving the equation

$$
(1-T) \cdot L[L(I)]+T \cdot L(I)=0
$$

where $T$ is a tension factor between 0 and 1 , and $L$ indicates the Laplacian operator (see Wessel and Smith, 1991). A tension factor of 0 yields the minimum-curvature solution, which can produce minima and maxima away from constrained values. With a value of 1 , no minima or maxima occur away from control points. A tension factor of 1.0 is preferred because it avoids introduction of extreme values not constrained by data (Fig. 3). Figure 4 presents a closeup view of the Kachchh region.

The intensity maps reveal several interesting features. The event was felt only lightly at the higher-elevation cities on Deccan lavas throughout central and southern India. Away from the Kachchh region, intensities were clearly amplified significantly in areas that are along rivers, within deltas, or on coastal alluvium. One example is the Narmada River Valley in the province of Madhya Pradesh, where MMI values as high as VI were reached at distances of $>600 \mathrm{~km}$. Significant site effects were also observed within Mumbai (Bombay). Most of the city experienced shaking at the MMI $\mathrm{V}$ level, but intensities up to VI-VII were reached at areas built on landfill in southern and central Mumbai as well as along Bombay Harbor.

Interesting features can be seen in the intensity distribution within the Kachchh region as well. The most heavily damaged villages are concentrated toward the western edge of the inferred fault, suggesting substantial western directivity from the epicenter. Some of the largest mud volcanos were also documented in this region (Tuttle et al., 2002). Significant sediment-induced amplification is also suggested at a number of locations around the Gulf of Kachchh, including towns immediately south of the epicenter and many of the villages on mudflats around the gulf.

The distribution of intensities in Kachchh are quite consistent with the spatial extent of liquefaction features as described by Tuttle et al. (2001, 2002). In northern Kachchh, the correspondence is not coincidental, as observations of liquefaction were used to assign some of the MMI values in some unpopulated areas. No liquefaction was observed in southwestern Kachchh, however, and the low MMI values in this region were assigned based on relatively light damage in this area. 
Table 1

Bhuj Earthquake Intensities

\begin{tabular}{|c|c|c|c|c|c|}
\hline Location & Lat. & Long. & MMI & Report & Source \\
\hline Adhoi, Gujarat & 23.400 & 70.513 & $9-10$ & Total devastation & Kutchinfo.com \\
\hline Adipur, Gujarat & 23.082 & 70.066 & $9-10$ & Total devastation & Zee News \\
\hline Ahemadabad, Gujarat & 23.043 & 72.578 & 7 & Some damage & The Indian Express \\
\hline Ahemadabad, Gujarat & 23.030 & 72.577 & 7 & Damage to mosque, bridge & (several) \\
\hline Ahemadabad, Gujarat & 23.009 & 72.590 & $7-8$ & Several high-rise buildings collapsed & (several) \\
\hline Ahemadabad, Gujarat & 23.009 & 72.568 & $7-8$ & Damage to soft-story high-rise buildings & Outlook, Times of India \\
\hline Ahemadabad, Gujarat & 23.050 & 72.577 & 6 & Walls slightly cracked & Zee News, Asian Age \\
\hline Ahemadabad, Gujarat & 23.058 & 72.564 & $7-8$ & Water table rose $2.5 \mathrm{~cm}$ & Times of India \\
\hline Ahemadabad, Gujarat & 23.030 & 72.551 & $7-8$ & Several high-rise buildings collapsed & (several) \\
\hline Ajmer, Rajasthan & 26.270 & 74.420 & 6 & Buildings cracked & The Hindu \\
\hline Akola, Maharashtra & 20.420 & 77.020 & 3 & Felt lightly, duration estimated & Sandhyanand \\
\hline Allahabad, Uttar Pradesh & 25.280 & 81.540 & 3 & Felt, many dizzy & The Hindu \\
\hline Amravati, Maharashtra & 20.560 & 77.480 & 3 & Felt lightly, duration estimated & Sandhyanand \\
\hline Amreli District, Gujarat & 21.360 & 71.150 & $7-8$ & 190 "Pucca" buildings destroyed & Kutchinfo.com \\
\hline Anand District, Gujarat & 22.320 & 73.000 & $6-7$ & Some buildings collapsed, many damaged & Kutchinfo.com \\
\hline Anjar, Gujarat & 23.117 & 70.019 & $10-11$ & Most old buildings leveled & Asian Age, Zee News \\
\hline Ayyampettai, Tamil Nadu & 10.902 & 79.182 & 3 & Felt & The Hindu \\
\hline Badin, Sindh (Pakistan) & 24.663 & 68.838 & $8-9$ & $\begin{array}{l}\text { Water emitted from cracks } \\
\text { Building damage }\end{array}$ & The Dawn \\
\hline Bagathala, Gujarat & 22.847 & 70.717 & $8-9$ & Most buildings damaged or destroyed & Asian Age \\
\hline Bahawalpur, Punjab (Pakistan) & 29.391 & 71.699 & $6-7$ & Buildings cracked & The Dawn \\
\hline Bajana, Gujarat & 23.118 & 71.768 & 8 & New springs & Times of India \\
\hline Bakhasar, Rajasthan & 24.430 & 71.090 & $7-8$ & Several buildings collapsed & The Indian Express \\
\hline Balamba, Gujarat & 22.716 & 70.436 & $8-9$ & Most buildings damaged or destroyed & Zee News \\
\hline Bangalore, Karnataka & 12.958 & 77.583 & $3-4$ & Felt widely, people ran outside & The Hindu \\
\hline Bangladesh, Bangladesh & 22.350 & 91.830 & 3 & Felt, western and central regions & 123india.com \\
\hline Bapatla, Andhra Pradesh & 15.905 & 80.466 & 3 & Felt & The Hindu \\
\hline Beraja, Gujarat & 22.986 & 69.600 & $5-6$ & Cracks in buildings & Panjokutch.com \\
\hline Bhachau, Gujarat & 23.287 & 70.352 & $9-10$ & Most buildings destroyed & $\begin{array}{l}\text { Zee News } \\
\text { Kutchinfo.com }\end{array}$ \\
\hline Bhadreshwar, Gujarat & 22.916 & 69.891 & $8-9$ & Many buildings severely damaged & Kutchinfo.com \\
\hline Bharuch, Gujarat & 21.719 & 72.971 & $7-8$ & Several buildings damaged & Kutchinfo.com \\
\hline Bhavnagar District, Gujarat & 21.460 & 72.110 & 7 & Many "pucca" buildings destroyed & Kutchinfo.com \\
\hline Bhilwara, Rajasthan & 25.210 & 74.400 & 6 & Buildings cracked & The Hindu \\
\hline Bhubaneshwar, Orissa & 20.150 & 85.520 & 3 & Felt & Pragativadi \\
\hline Bhuj, Gujarat & 23.245 & 69.662 & $11-12$ & Widespread devastation, pipes destroyed & (several) \\
\hline Bhujpur, Gujarat & 22.867 & 69.635 & $7-8$ & Ground level sunk (liquefaction) & Panjokutch.com \\
\hline Bidada, Gujarat & 22.900 & 69.463 & $6-7$ & Light damage & Panjokutch.com \\
\hline Bidar, Karnataka & 17.570 & 77.390 & 3 & Felt & Indiaexpress.com \\
\hline Buldhana, Maharashtra & 20.320 & 76.140 & 3 & Felt lightly, duration estimated & Sandhyanand \\
\hline Butchireddipalem, Andhra Pradesh & 14.531 & 79.884 & 3 & Felt & The Hindu \\
\hline Chandigarh, Chandigarh & 30.420 & 76.540 & 3 & Many people felt giddy/nauseous & ASC report \\
\hline Chennai, Tamil Nadu & 13.040 & 80.170 & 4 & Kitchen utensils fell & The Hindu \\
\hline Chhasra, Gujarat & 22.969 & 69.816 & $8-9$ & $80 \%$ Houses totally damaged & Panjokutch.com \\
\hline Chidambaram, Andhra Pradesh & 11.399 & 79.762 & 3 & Felt & The Hindu \\
\hline Chitrod, Gujarat & 23.40 & 70.70 & 8 & Damage to temple & INTACH field rep. \\
\hline Cuddalore, Andhra Pradesh & 11.753 & 79.769 & 3 & Felt & The Hindu \\
\hline Dalauda, Madhya Pradesh & 23.934 & 75.099 & NF & Not felt by observer ground & ASC report \\
\hline Deesa, Gujarat & 24.25 & 72.167 & $7-8$ & Church collapsed & Indiaexpress.com \\
\hline Deshalpur, Gujarat & 23.735 & 70.681 & $6-7$ & Light damage to village & Panjokutch.com \\
\hline Dholavira, Gujarat & 23.438 & 66.766 & 9 & Archeological Society building destroyed & Express \\
\hline Dhori, Gujarat & 23.438 & 66.766 & $9-10$ & Fissures, sand blows, sand craters & Reuters, Zee News \\
\hline Dhrandadhra, Gujarat & 22.991 & 71.467 & 8 & New springs & Times of India \\
\hline Dhrol, Gujarat & 22.574 & 70.407 & 8 & Heavy damage & www.xtechindia.com \\
\hline Dhule, Maharashtra & 20.580 & 74.470 & 5 & Felt strongly & Kesri \\
\hline Dudhai, Gujarat & 23.318 & 70.134 & $9-10$ & Most buildings destroyed & Times of India \\
\hline Dwarka, Gujarat & 22.247 & 68.965 & 8 & Temples damaged & Times of India \\
\hline Gandhidham, Gujarat & 23.074 & 70.131 & $9-10$ & Many high-rise building collapsed & Star News, AP \\
\hline Gandhinagar, Gujarat & 23.296 & 72.635 & 8 & Water table rose $2.5 \mathrm{~cm}$ & Times of India \\
\hline Ganeshpuri-Vajreshwari Maharashtra & 19.492 & 72.998 & 8 & Change in hot springs temp., level & Star News \\
\hline Ghotki, Sindh (Pakistan) & 28.000 & 69.325 & 3 & "Brief spell of earthquake" & The Dawn \\
\hline
\end{tabular}


Table 1

Bhuj Earthquake Intensities (continued)

\begin{tabular}{|c|c|c|c|c|c|}
\hline Location & Lat. & Long. & MMI & Report & Source \\
\hline Goa (entire), Goa & 14.200 & 74.000 & $3-4$ & People fled outside, articles rattled & Sandhyanand \\
\hline Gundala, Gujarat & 22.901 & 69.752 & $9-10$ & Heavy damage, all houses destroyed & Kutchinfo.com \\
\hline Guntur, Andhra Pradesh & 16.294 & 80.444 & 3 & Felt & The Hindu \\
\hline Gwalior, Madhya Pradesh & 26.140 & 78.100 & $4-5$ & Felt strongly, utensils fell & Sandhyanand \\
\hline Halvad, Gujarat & 23.017 & 71.174 & 8 & New springs & Times of India \\
\hline Haryana (entire) & 30.300 & 74.600 & 3 & Felt for "around $20 \mathrm{sec} . "$ & Sandhyanand \\
\hline Himachal Pradesh & 32.290 & 76.100 & 3 & Felt for "around $20 \mathrm{sec} . "$ & Sandhyanand \\
\hline Hoshangabad, Madhya Pradesh & 22.460 & 77.450 & $4-5$ & Felt strongly, utensils fell & Sandhyanand \\
\hline Hyderabad, Sindh (Pakistan) & 25.250 & 68.380 & $7-8$ & Damage to buildings, dozens injured & The Dawn \\
\hline Hyderabad, Andhra Pradesh & 17.387 & 78.480 & $2-3$ & Felt only in tall buildings & The Hindu \\
\hline Jacobabad, Sindh (Pakistan) & 28.279 & 68.428 & 3 & "Brief spell of earthquake" & The Dawn \\
\hline Jaipur, Rajasthan & 26.893 & 75.790 & 6 & Some buildings cracked & (check) \\
\hline Jaiselmer, Rajasthan & 26.914 & 70.790 & 7 & Buildings cracked, damaged & The Indian Express \\
\hline Jalgaon, Maharashtra & 21.050 & 75.400 & 3 & Felt & Kesri \\
\hline Jalore, Rajasthan & 25.220 & 72.580 & 6 & Buildings cracked & The Hindu \\
\hline Jamnagar, Gujarat & 22.467 & 70.067 & 9 & Many buildings destroyed & $\begin{array}{l}\text { Zee News } \\
\text { Kutchinfo.com }\end{array}$ \\
\hline Jawaharnagar, Gujarat & 23.367 & 69.986 & 10 & Many buildings completely destroyed & Indian Express, AP \\
\hline Jhinjhuwada, Gujarat & 23.356 & 71.747 & 8 & New springs & Times of India \\
\hline Jodhpur, Rajasthan & 21.883 & 70.033 & $7-8$ & Collapse of building dome & The Indian Express \\
\hline Junagadh, Gujarat & 21.516 & 70.457 & $7-8$ & Many buildings destroyed & Kutchinfo.com \\
\hline Kabul (Afghanistan) & 34.561 & 69.083 & 3 & Felt & The Indian Express \\
\hline Kandla, Gujarat & 23.051 & 70.215 & 9 & Many buildings severely damaged & (several) \\
\hline Kandla Port Trust, Gujarat & 22.982 & 70.218 & 9 & $\begin{array}{l}\text { Several buildings collapsed } \\
\text { Piers damaged, widespread liquefaction }\end{array}$ & Times of India \\
\hline Kanpur, Uttar Pradesh & 26.280 & 80.240 & $3-4$ & Felt, furniture rattled & Indiaexpress.com \\
\hline Karachi, Sindh (Pakistan) & 24.510 & 67.040 & $5-6$ & Doors opened and closed, building cracks & ASC report \\
\hline Kathmandu (Nepal) & 27.734 & 85.282 & $3-4$ & Some reports of objects swinging & AFP \\
\hline Kera Badadia, Gujarat & 23.083 & 69.598 & 7 & All buildings damaged & Panjokutch.com \\
\hline Kerala & 10.0 & 76.25 & NF & Not felt & Indiaexpress.com \\
\hline Khadan, Sindh (Pakistan) & 24.492 & 68.987 & 9 & $6^{\prime \prime}$ cracks, sand/water emitted & The Dawn \\
\hline Khaipur, Sindh (Pakistan) & 27.280 & 68.440 & $5-6$ & Some damage & The Dawn \\
\hline Khangharpur, Gujarat & NL & NL & $8-9$ & $6^{\prime \prime}$ cracks, sand/water emitted & Reuters \\
\hline Kharaghodha Tank, Gujarat & 23.231 & 71.747 & 8 & New springs & Times of India \\
\hline Khavda, Gujarat & 23.840 & 69.720 & 9 & $\begin{array}{l}\text { Most buildings destroyed } \\
\text { Possible mud volcano }\end{array}$ & Times of India \\
\hline Kheda District, Gujarat & 22.450 & 72.450 & $6-7$ & Many buildings damaged & Kutchinfo.com \\
\hline Kolhapur, Maharashtra & 16.707 & 79.224 & 3 & Felt & Indiaexpress.com \\
\hline Kolkata, West Bengal & 22.340 & 88.240 & $3-4$ & Overhead fixtures swung & Star News, Sandhyanand \\
\hline Kota, Rajasthan & 25.178 & 75.835 & 6 & Railway station cracked & The Indian Express \\
\hline Kotdi-Roha, Gujarat & 23.136 & 69.255 & 9 & Two dead, heavy damage to KVO houses & Panjokutch.com \\
\hline Kotri, Sindh (Pakistan) & 25.220 & 68.220 & $4-5$ & 25 women fainted, strong shaking & The Dawn \\
\hline Koyna, Maharashtra & 17.398 & 73.767 & $3-4$ & Felt for "around $40 \mathrm{sec} . "$ & Sandhyanand \\
\hline Kuda, Gujarat & 23.113 & 71.385 & 8 & New springs & Times of India \\
\hline Kumbakakonam, Tamil Nadu & 10.961 & 79.182 & $4-5$ & People ran, strongly felt & The Hindu \\
\hline Lahore, Punjab (Pakistan) & 31.542 & 74.399 & $4-5$ & Reported as "severe" & The Dawn \\
\hline Larkana, Sindh (Pakistan) & 27.330 & 68.150 & 3 & "Brief spell of earthquake" & The Dawn \\
\hline Lodhai, Gujarat & 23.402 & 69.880 & $10-11$ & Most buildings distroyed & Midday \\
\hline Lucknow, Uttar Pradesh & 26.550 & 80.590 & $3-4$ & Furniture rattled & Indiaexpress.com \\
\hline Luna, Gujarat & 23.714 & 69.252 & $8-9$ & Water jet observed & Kutchinfo.com \\
\hline Machilipatnam Andhra Pradesh & 16.187 & 81.135 & 3 & Felt & The Hindu \\
\hline Maheshwari, Madhya Pradesh & 22.110 & 75.370 & 6 & Maheshwari fort cracked & Sandhyanand \\
\hline Maliya, Gujarat & 23.093 & 70.748 & 8 & New springs, water levels increased & Times of India \\
\hline Mandsaur, Madhya & 23.030 & 75.080 & $5-6$ & Household articles knocked down & ASC report \\
\hline Mandvi, Gujarat & 22.834 & 69.343 & 9 & Many buildings collapsed, bridges damaged & Times of India \\
\hline Matiari, Sindh (Pakistan) & 25.596 & 68.443 & $6-7$ & Wall collapse & The Dawn \\
\hline Mehsana District, Gujarat & 23.420 & 72.370 & $7-8$ & 12 "pucca" buildings destroyed & Kutchinfo.com \\
\hline Mirpurkhas, Sindh Pakistan & 25.522 & 69.010 & $7-8$ & Walls and roofs collapsed & The Dawn \\
\hline Mithi, Sindh (Pakistan) & 24.732 & 69.792 & $7-8$ & Walls and roofs collapsed & The Dawn \\
\hline Modhera, Gujarat & 23.587 & 72.132 & $6-7$ & Sun Temple damaged & Indya.com \\
\hline Morbi, Gujarat & 22.811 & 70.827 & 8 & Many buildings severely damaged & ASC report \\
\hline
\end{tabular}


Table 1

Bhuj Earthquake Intensities (continued)

\begin{tabular}{|c|c|c|c|c|c|}
\hline Location & Lat. & Long. & MMI & Report & Source \\
\hline Mota Asambia, Gujarat & 22.968 & 69.447 & 10 & Most buildings destroyed & Kutchinfo.com \\
\hline Multan, Punjab (Pakistan) & 31.452 & 71.455 & $6-7$ & Buildings cracked & The Dawn \\
\hline Mumbai (Andheri) Maharashtra & 19.123 & 72.912 & 5 & People fled outside & ASC report \\
\hline Mumbai (Antop Hill) Maharashtra & 19.028 & 72.843 & 6 & Buildings cracked & Sandhyanand \\
\hline Mumbai (Bandra) Maharashtra & 19.058 & 72.836 & $3-4$ & Felt distinctly & ASC report \\
\hline Mumbai (Colaba) Maharashtra & 18.907 & 72.809 & 6 & People fled into streets & Sandhyanand \\
\hline Mumbai (Crawford Market) Maharashtra & 18.950 & 72.829 & 6 & Buildings cracked & ASC report \\
\hline Mumbai (Dahisar) Maharashtra & 19.258 & 72.837 & 5 & Windows rattled & ASC report \\
\hline Mumbai (Kurla) Maharashtra & 19.076 & 72.912 & 6 & Buildings cracked & Kesri \\
\hline Mumbai (Malad) Maharashtra & 19.183 & 72.832 & $4-5$ & Felt strongly & ASC report \\
\hline Mumbai (Mankhurd) Maharashtra & 19.050 & 72.931 & 6 & Buildings cracked & Sandhyanand \\
\hline Mumbai (Mazegaon) Maharashtra & 18.968 & 72.841 & 6 & Building cracked & ASC report \\
\hline Mumbai (Mumbai Central) Maharashtra & 18.993 & 72.827 & 6 & Glassware broke, fixtures swung & ASC report \\
\hline Mumbai (Navynagar) Maharashtra & 18.912 & 72.813 & $5-6$ & People fled into streets & Sandhyanand \\
\hline Mumbai (Vikhroli) Maharashtra & 19.096 & 72.929 & $5-6$ & Building cracked & Sandhyanand \\
\hline Mumbai (Wadala) Maharashtra & 19.028 & 72.843 & $6-7$ & Section of fire station collapsed & Sandhyanand \\
\hline Mumbai (Worli) Maharashtra & 19.015 & 72.819 & 6 & Felt strongly, building damage & Times of India Sandhyanand \\
\hline Muzzafarnagar Uttar Pradesh & 29.280 & 77.440 & 3 & Felt by many & Sandhyanand \\
\hline Nakhatrana, Gujarat & 23.352 & 69.258 & 9 & Sand blows, fountains & Times of India \\
\hline Nalasopara, Maharashtra & 19.417 & 72.782 & 5 & Household objects shaken & ASC report \\
\hline Nanded, Maharashtra & 19.090 & 77.270 & 3 & Felt lightly, duration estimated & Sandhyanand \\
\hline Nandiad, Gujarat & 22.687 & 72.854 & 7 & Buildings visibly shaken & BBC Talking Point \\
\hline Nandurbar, Maharashtra & 21.230 & 74.190 & 3 & Felt & Kesri \\
\hline Nashik, Maharashtra & 20.001 & 73.781 & $6-7$ & Several buildings damaged & Sandhyanand, ASC report \\
\hline Naushahro Firoz, Sindh Pakistan & 26.848 & 68.122 & $6-7$ & Buildings damaged & The Dawn \\
\hline Navlakhi, Gujarat & 22.969 & 70.464 & 8 & Railway tracks submerged & Asian Age \\
\hline & & & & Liquefaction & Times of India \\
\hline Navsari, Gujarat & 20.954 & 72.919 & $7-8$ & 98 "pucca" buildings collapsed & Kutchinfo.com \\
\hline Nawabshah, Sindh Pakistan & 26.236 & 68.394 & $7-8$ & Buildings damaged & The Dawn \\
\hline New Delhi, NCT & 28.380 & 77.120 & $3-4$ & Felt, overhead fixtures swung & $N D T V$ \\
\hline Neyvel, Andhra Pradesh & 11.607 & 79.491 & 3 & Felt & The Hindu \\
\hline Nindo Shahr, Sindh & 24.638 & 69.037 & I & Several injured & The Dawn \\
\hline Noida, Uttar Pradesh & 28.605 & 77.260 & $3-4$ & Overhead fixtures swung & ASC report \\
\hline Okha, Gujarat & 22.462 & 69.061 & 8 & Port facilities slightly damaged & Sandhyanand \\
\hline Osmanabad, Maharashtra & 18.080 & 76.060 & 3 & Felt lightly, duration estimated & Sandhyanand \\
\hline Palanpur, Gujarat & 24.171 & 72.430 & $7-8$ & $\begin{array}{l}\text { Many buildings collapsed } \\
\text { Old bridge damaged }\end{array}$ & (several) \\
\hline Pali, Rajasthan & 25.460 & 73.250 & 6 & Buildings cracked & The Hindu \\
\hline Papanad, Tamil Nadu & 10.536 & 79.282 & 3 & Felt & The Hindu \\
\hline Papanasam, Tamil Nadu & 10.922 & 79.270 & 3 & Felt & The Hindu \\
\hline Patan, Gujarat & 23.874 & 72.109 & $7-8$ & Many buildings collapsed & Kutchinfo.com \\
\hline Patdi, Gujarat & 23.197 & 71.792 & 8 & New springs & The Times of India \\
\hline Patna, Bihar & 25.370 & 85.130 & 3 & Felt & The Tribune \\
\hline Peshawar, NWFP Pakistan & 33.276 & 71.860 & 3 & Felt & The Dawn \\
\hline Pokhran, Rajasthan & 26.550 & 71.580 & 6 & Buildings cracked & Indian Express \\
\hline Pondicherry, (UT) & 11.933 & 79.835 & $4-5$ & Celebrations disrupted, utensils fell & The Hindu \\
\hline Ponnuru, Andhra Pradesh & 16.067 & 80.560 & 3 & Felt & The Hindu \\
\hline Porbander, Gujarat & 21.644 & 69.603 & $7-8$ & Many buildings destroyed & Zee News, Kutchinfo.com \\
\hline Pune, Camp, Maharashtra & 18.310 & 73.550 & 5 & Furniture, windows rattled & ASC report \\
\hline Pune, Hadapsar Maharashtra & 18.503 & 73.887 & NF & Observers were on ground floor & ASC report \\
\hline Pune Lohegaon-Vimannagar Maharashtra & 18.589 & 73.898 & $4-5$ & Windows and furniture rattled & ASC report \\
\hline Pune, Lullanagar Maharashtra & 18.496 & 73.859 & 3 & Felt & ASC report \\
\hline Pune, Sassoon Road Maharashtra & 18.533 & 73.853 & $4-5$ & Household articles, furniture shook & ASC report \\
\hline Punjab (entire) & 30.400 & 75.500 & 3 & Felt for "around $20 \mathrm{sec} . "$ & Sandhyanand \\
\hline Quetta, Baluchistan (Pakistan) & 30.309 & 67.019 & 3 & Felt & The Dawn \\
\hline Radhanpur, Gujarat & 23.841 & 71.603 & 6 & Concrete water tanks swayed & USGS report \\
\hline Rajkot, Gujarat & 22.301 & 70.801 & $7-8$ & Many buildings collapsed & Zee News Times of India \\
\hline Rapar, Gujarat & 23.576 & 70.641 & 10 & Most buildings destroyed & (several) \\
\hline Ratnal, Gujarat & 23.194 & 69.870 & 10 & Most buildings destroyed & Kutchinfo.com \\
\hline Rohri, Sindh (Pakistan) & 27.410 & 68.570 & 3 & "Brief spell of earthquake" & The Dawn \\
\hline Salem, Tamil Nadu & 11.390 & 78.120 & $\mathrm{NF}$ & Not felt & ASC report \\
\hline
\end{tabular}


Table 1

Bhuj Earthquake Intensities (continued)

\begin{tabular}{|c|c|c|c|c|c|}
\hline Location & Lat. & Long. & MMI & Report & Source \\
\hline Samakhiali, Gujarat & 23.329 & 70.587 & 9 & Water flooded salt pans ground cracking & Times of India \\
\hline Sanghar, Sindh (Pakistan) & 26.050 & 68.937 & $6-7$ & Buildings damaged & The Dawn \\
\hline Shikarpur, Sindh (Pakistan) & 27.965 & 68.635 & 3 & "Brief spell of earthquake" & The Dawn \\
\hline Shillong, Meghalaya & 25.340 & 91.560 & 3 & Felt & Sandhyanand \\
\hline Sirohi, Rajasthan & 24.530 & 72.540 & 6 & Buildings cracked & The Hindu \\
\hline Sukhpur, Gujarat & 23.232 & 69.600 & $11-12$ & 10-yr old "flung into air" & The Asian Age \\
\hline Sukkur, Sindh (Pakistan) & 27.693 & 68.845 & 3 & "Brief spell of earthquake" & The Dawn \\
\hline Suraj Bari, Gujarat & 23.207 & 70.703 & $8-9$ & Serious cracks in land bridge & Times of India \\
\hline Surat, Gujarat & 21.193 & 72.822 & $7-8$ & $\begin{array}{l}\text { A few high-rise buildings collapsed } \\
\text { Nuclear reactor fba not triggered, } \\
\text { Indicating shaking less than } 0.1 \mathrm{~g}\end{array}$ & (several) \\
\hline Surendranagar, Gujarat & 22.706 & 71.678 & 8 & Many old buildings destroyed & Star News \\
\hline Suvi, Gujarat & 23.618 & 70.483 & $9-10$ & Damage to dam & IIT Kanpur \\
\hline Tada, Andhra Pradesh & 13.586 & 80.030 & 3 & Felt & The Hindu \\
\hline Tadepalli, Andhra Pradesh & 16.477 & 80.601 & 3 & Felt & The Hindu \\
\hline Talhar, Sindh (Pakistan) & 24.894 & 68.806 & I & Two injured & The Dawn \\
\hline Tando Allah Yar, Sindh (Pakistan) & 25.459 & 68.716 & $6-7$ & Wall collapse, 1 dead & The Dawn \\
\hline Tarapur, Maharashtra & 19.880 & 73.688 & $5-6$ & Reactors did not shut down & ASC report \\
\hline Thane, Maharashtra & 19.120 & 73.020 & 4 & Felt strongly, esp. on upper floors & ASC report \\
\hline Thatta, Sindh (Pakistan) & 24.751 & 67.923 & $6-7$ & 3 motorbike riders lost control & The Dawn \\
\hline Thiruvaiyaru, Tamil Nadu & 10.884 & 79.098 & $4-5$ & Some objects fell in market & The Hindu \\
\hline Tivim, Goa & 15.598 & 73.831 & $\mathrm{NF}$ & Not felt & ASC report \\
\hline Tonk District Rajasthan & 26.110 & 75.500 & 6 & Buildings cracked & The Hindu \\
\hline Udaipur, Rajasthan & 27.420 & 75.330 & 7 & Serious damage to factory & Sandhyanand \\
\hline Ujjain, Madhya Pradesh & 23.090 & 77.430 & $4-5$ & Felt strongly, utensils fell & Sandhyanand \\
\hline Unchahar, Uttar Pradesh & 25.857 & 81.630 & 3 & Many people felt giddy/naseuos & ASC report \\
\hline Unnao, Uttar Pradesh & 26.480 & 80.430 & $3-4$ & Furniture shook & Indiaexpress.com \\
\hline Vadala, Gujarat & 22.918 & 69.850 & $7-8$ & Most houses damaged, few collapsed & Panjokutch.com \\
\hline Vadodara, Gujarat & 22.303 & 73.187 & 6 & Minor damage to buildings & ASC report \\
\hline Valsad, Gujarat & 20.611 & 72.924 & 7 & Many buildings damaged & Kutchinfo.com \\
\hline Vidisha, Madhya Pradesh & 23.320 & 77.510 & $4-5$ & Felt strongly, utensils fell & Sandhyanand \\
\hline Vijayawada, Andhra Pradesh & 16.517 & 80.635 & 3 & Felt & ASC report \\
\hline Vishakhapatnam, Andhra Pradesh & 17.728 & 83.304 & 3 & Felt & The Hindu \\
\hline Vondh, Gujarat & 23.301 & 70.397 & 10 & $\begin{array}{l}\text { Most old buildings collapsed } \\
\text { Some newer structures badly damaged }\end{array}$ & Zee News, AP \\
\hline Wankaner, Gujarat & 22.612 & 70.934 & 7 & Fallen masonry & Times of India \\
\hline
\end{tabular}

Location: city, province, and country (if not India).

\section{Predicted Ground Motions}

Although the Bhuj earthquake was not recorded by strong-motion instruments, it was well recorded at teleseismic distances (e.g., Yagi and Kikuchi, 2001). We use a simplified source model determined from instrumental data to predict ground motions at local and regional distances using the finite-fault method of Beresnev and Atkinson (1997). This analysis is complicated by the fact that neither the ground motions nor the fault parameters are well constrained. We therefore seek to investigate only the general consistency between the inferred and predicted ground motions.

Our fault model is based on the moment, strike, dip, and rake determined by the U.S. Geological Survey, assuming a south-dipping fault plane (see Fig. 1). We use a moment magnitude of 7.6, consistent with finite-fault inversions from teleseismic data (Yagi and Kikuchi, 2001). We assume a rupture length of $50 \mathrm{~km}$ based on preliminary aftershock results (e.g., Horton et al., 2001) and use an initial fault depth of $9 \mathrm{~km}$ based on preliminary analysis of geodetic data. Finally, we use a smooth-rupture model in which the average slip is determined from the moment and fault area. We calculate ground motions for hard-rock-site conditions $(\kappa=0.005$; shear-wave velocity $=3.7 \mathrm{~km} / \mathrm{sec})$ and consider the issue of site response only in a qualitative manner. No crustal amplification is applied to the predictions. For our attenuation model, we use the results of Singh et al. (1999) for $L g$ attenuation in India: $Q=508 f^{0.48}$. We use a geometrical spreading function that includes an $r^{-1}$ decay from 0 to $50 \mathrm{~km}$ and an $r^{-0.5}$ decay beyond $50 \mathrm{~km}$, a slightly simpler form of the function assumed by Singh et al. (1999).

In the Beresnev and Atkinson (1997) approach, a rupture is simulated using fault-plane subelements, each of which is treated as a point source with a spectral shape constrained to have an $\omega^{2}$ shape. The method is attractive for this application because of its computational ease and because there are few model parameters to be assigned. It is 


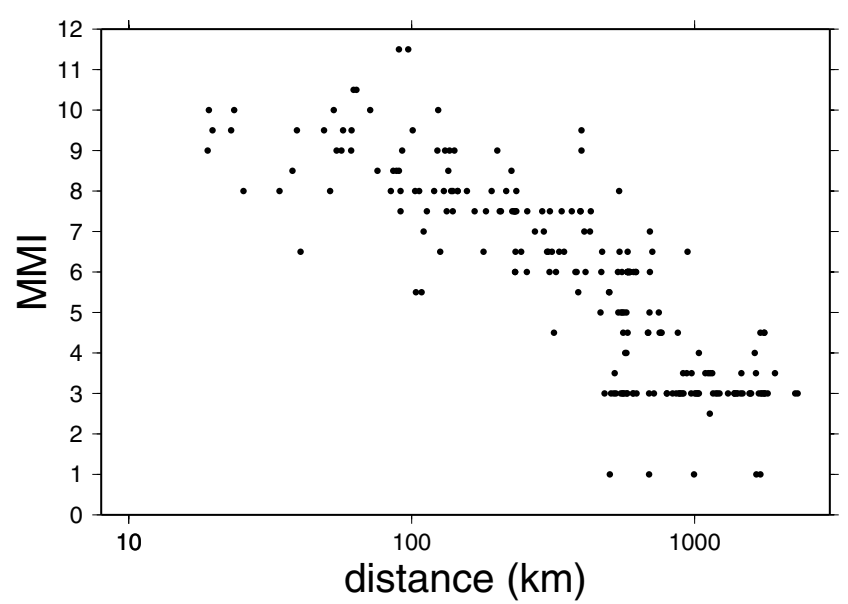

Figure 2. Inferred MMI values for the Bhuj earthquake are shown as a function of distance from the source. To estimate source distance, we calculate the nearest distance from each point to the "pseudo-fault" shown in Figure 1.

limited in its ability to model the time-domain characteristics of low-frequency ground motions, but we consider it likely that the damage from the Bhuj earthquake is primarily controlled by relatively high-frequency shaking.

The most important free parameter in this method is the "strength parameter," $S_{f}$, which is related to the maximum slip velocity, $v_{m}$, according to

$$
v_{m}=0.618 y(\Delta \sigma) S_{f} /(\rho \beta)
$$

where $\beta$ is the shear-wave velocity, $y$ is the rupture-propagation velocity as a fraction of $\beta, \Delta \sigma$ is the subevent stress drop, and $\rho$ is density (Beresnev and Atkinson, 2001). Although rupture velocity can vary along strike, the formulation of Beresnev and Atkinson (2002) includes only a single value of $S_{f}$ for each rupture model. As discussed by Beresnev and Atkinson (2001), the amplitude of high-frequency radiation depends strongly on $S_{f}$. $S_{f}$ was found to vary between 1.0 and 2.4 for a wide range of earthquakes in eastern and western North America. In our application, the depth of faulting is another unknown. We therefore calculate peak ground acceleration (PGA) for a suite of possible rupture models with varying depths and strength parameters. We vary the depth to the upper edge of the rupture between 3 and $9 \mathrm{~km}$ and vary the strength factor between 1.4 and 2.0.

Figure $5 \mathrm{a}, \mathrm{b}$ shows the predicted ground motions for hard-rock-site conditions as a function of distance for models in which strength factor and depth are varied, respectively. We conclude that predicted ground motions are more sensitive to the strength factor than to depth. Unfortunately, it is difficult to constrain the strength parameter (or, equivalently, the slip velocity.) For North America, its average value is 1.6 (Beresnev and Atkinson, 2002). We find that a strength factor close to this value (1.8) predicts a PGA of $10 \% \mathrm{~g}$ at the distance of Ahmedabad, consistent with the sin- gle strong-motion recording that was released in the aftermath of the earthquake (Fig. 5a). According to available reports, this instrument was located on a hard-rock site. There is some question, however, whether or not this instrument functioned properly (S. K. Singh, personal comm., 2002).

S. K. Singh et al. (unpublished manuscript) present the broadband data recorded from the Bhuj earthquake at distances of 565 to well $>1000 \mathrm{~km}$. Their peak acceleration values are also shown on Figure 5a for stations within 1350 $\mathrm{km}$. These more-distant recordings appear to be consistent with a somewhat lower value of $S_{f}$, although at large distances, the ground-motion curves are increasingly controlled by attenuation rather than $S_{f}$. We therefore adopt a preferred value of 1.6. With the other assumed fault parameters, this predicts near-field hard-rock ground motions of approximately $80 \% g$. The value of $S_{f}$ is clearly quite uncertain, however, with other plausible values implying near-field ground motions approximately $20 \%$ higher and lower.

To compare predicted and estimated intensities, we convert predicted PGA to MMI by using the calibration established by Wald et al. (1999). It should be borne in mind that PGA (and thus, MMI) is predicted for rock sites and that MMI on soil will be as much as 1-2 units larger than on rock (Hough et al., 2000; Atkinson, 2001). Although it is clearly difficult to compare data and models in cases where both are uncertain, we find that the predicted ground motions are able to match several salient features of the shaking distribution determined from MMI data. In both data and models, we find the highest shaking to the north and northwest of the epicenter and relatively low shaking to the southwest of the epicenter, as shown in Figure 6a.

For a wide range of strength factors, the model corroborates the macroseismic observation that potentially damaging ground motions can occur at distances of at least several hundred kilometers from the source. That is, PGAs on the order of $5 \% g$ generally correspond with the threshold of damage (e.g., Wald et al., 1999). Values near or above this value are predicted (for our preferred model) to a distance of nearly $400 \mathrm{~km}$. Moreover, because site response at soil sites can typically elevate MMI values by 1-2 units (e.g., Hough et al., 2000; Atkinson, 2001), the predicted hard-rock ground motions (Fig. 6a) are high enough to cause damage at soft-sediment sites especially, over the extent of the MMI IV region in these figures. (Values near or above $2.5 \% \mathrm{~g}$ are predicted for distances upward of $500 \mathrm{~km}$ in our preferred model.)

The residuals between observed intensities and those predicted on rock shown in Figure 7 are also interesting to consider. These are calculated simply by subtracting the predicted MMI values from those observed. We calculate residuals by using ground-motion predictions determined for $S_{f}=1.6$ and find that most values are between 1 and 3 MMI units. The distribution of residuals is generally consistent with expectations for site response, as especially high residuals are found at presumed sediment sites to the northeast and southeast of the rupture. Relatively low residuals are 


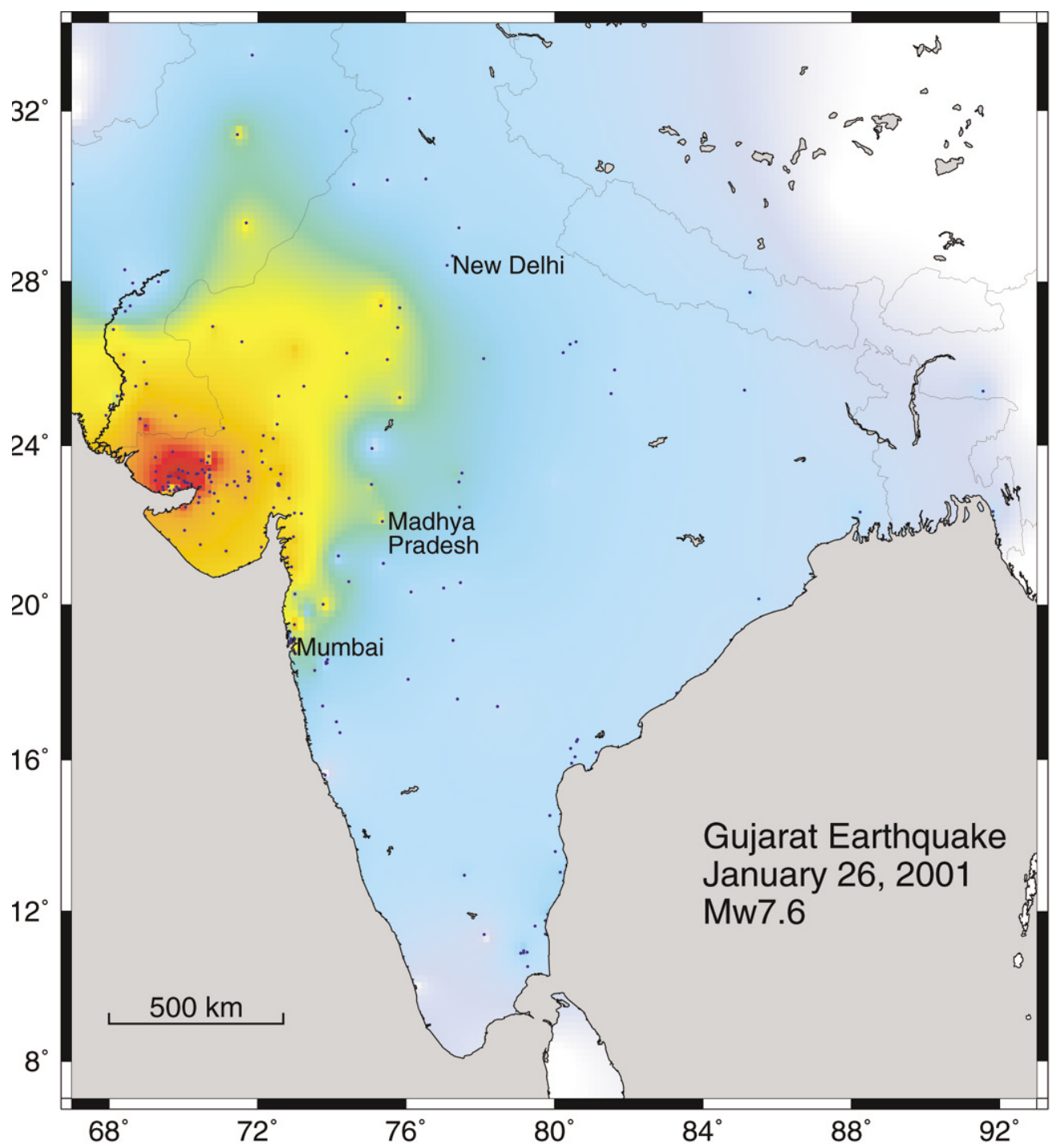

\begin{tabular}{|c|c|c|c|c|c|c|c|c|c|}
\hline $\begin{array}{c}\text { PERCEIVED } \\
\text { SHAKING }\end{array}$ & Not felt & Weak & Light & Moderate & Strong & Very strong & Severe & Violent & Extreme \\
\hline $\begin{array}{c}\text { POTENTIAL } \\
\text { DAMAGE }\end{array}$ & none & none & none & Very light & Light & Moderate & Moderate/Heavy & Heavy & Very Heavy \\
\hline PEAK ACC.(\%g) & $<.17$ & $\mathbf{. 1 7 - 1 . 4}$ & $\mathbf{1 . 4 - 3 . 9}$ & $\mathbf{3 . 9 - 9 . 2}$ & $\mathbf{9 . 2 - 1 8}$ & $18-34$ & $34-65$ & $65-124$ & $>124$ \\
\hline PEAK VEL.(cm/s) & $<0.1$ & $\mathbf{0 . 1 - 1 . 1}$ & $1.1-3.4$ & $3.4-8.1$ & $8.1-16$ & $\mathbf{1 6 - 3 1}$ & $\mathbf{3 1 - 6 0}$ & $\mathbf{6 0 - 1 1 6}$ & $>\mathbf{1 1 6}$ \\
\hline $\begin{array}{c}\text { INSTRUMENTAL } \\
\text { INTENSITY }\end{array}$ & $\mathbf{I}$ & II-III & $\mathbf{I V}$ & $\mathbf{V}$ & $\mathbf{V I}$ & VII & VIII & IX & X+ \\
\hline
\end{tabular}

Figure 3. Map of intensity distribution for the 2001 Bhuj earthquake determined using a smoothing parameter of 1.0. MMI values are constrained at approximately 200 locations indicated with small circles. Colors reflect MMI values according to scale shown at bottom of figure. Ground-motion parameters corresponding to each MMI value are from recent earthquakes in California (Wald et al., 1999).

also found at locations to the southeast, which lie on Deccan lavas.

A coherent band of low residuals is also observed along the Indus River in Pakistan. Regional geological maps indicate that these sites should be alluvial. However, we speculate that the relatively low ground motions in this region may reflect path rather than site effects. That is, the active plate boundary west and northwest of Gujarat will likely disrupt coherent $L g$ wave propagation, which will give rise to a higher apparent attenuation and lower intensities (Kennett, 1989; Hanks and Johnston, 1992). Considering the spatial distribution of residuals, we speculate that the true regional attenuation curve might be somewhat steeper than that predicted by Singh et al. (1999). 


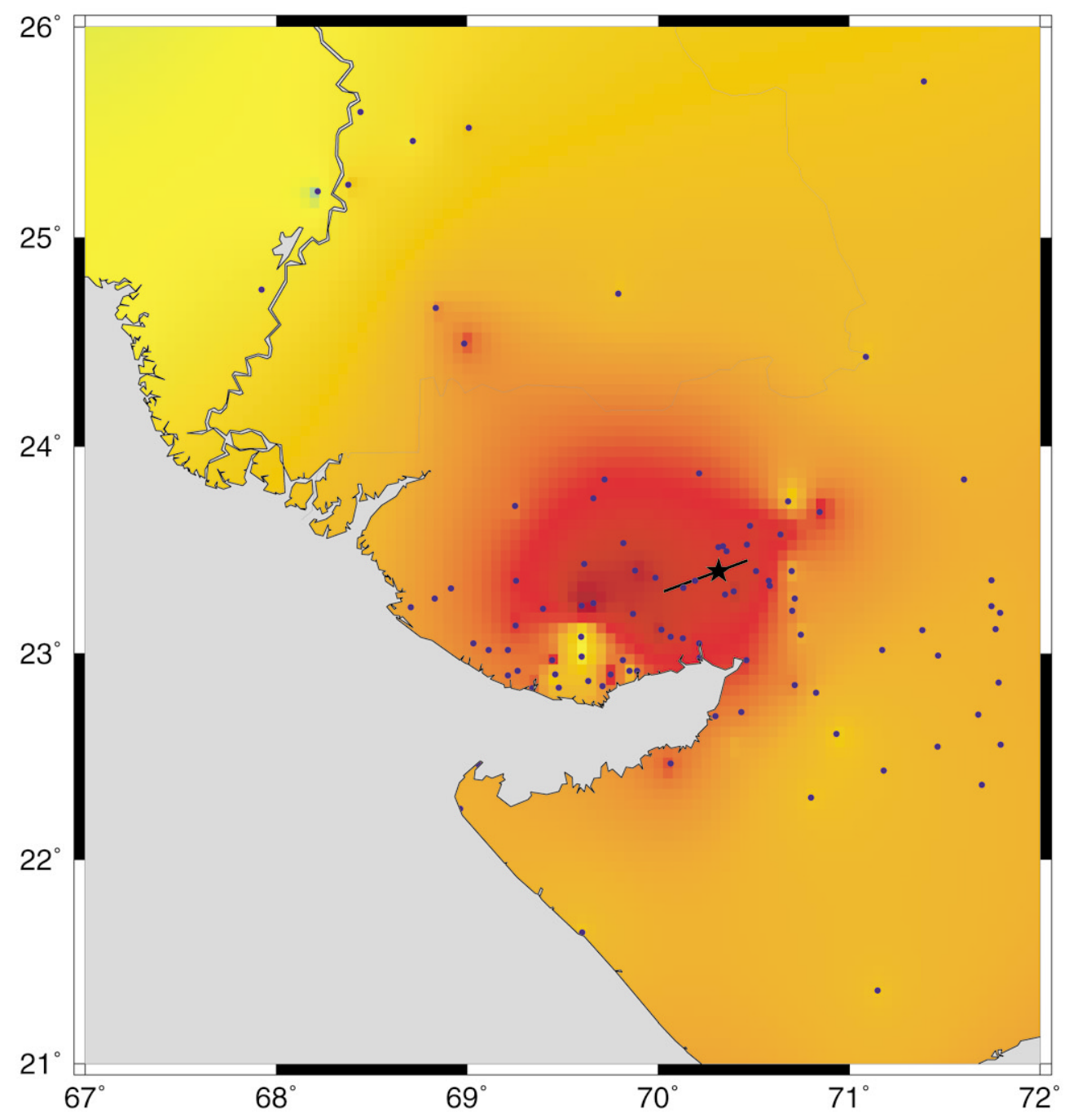

\begin{tabular}{|c|c|c|c|c|c|c|c|c|c|}
\hline $\begin{array}{c}\text { PERCEVED } \\
\text { SHAKING }\end{array}$ & Not felt & Weak & Light & Moderate & Strong & Very strong & Severe & Violent & Extreme \\
\hline $\begin{array}{c}\text { POTENTIAL } \\
\text { DAMAGE }\end{array}$ & none & none & none & Very light & Light & Moderate & Moderate/Heavy & Heavy & Very Heavy \\
\hline PEAK ACC.(\%g) & $<.17$ & $\mathbf{. 1 7 - 1 . 4}$ & $\mathbf{1 . 4 - 3 . 9}$ & $\mathbf{3 . 9 - 9 . 2}$ & $\mathbf{9 . 2 - 1 8}$ & $18-34$ & $34-65$ & $65-124$ & $>124$ \\
\hline PEAK VEL.(cm/s) & $<0.1$ & $\mathbf{0 . 1 - 1 . 1}$ & $1.1-3.4$ & $3.4-8.1$ & $8.1-16$ & $\mathbf{1 6 - 3 1}$ & $\mathbf{3 1 - 6 0}$ & $\mathbf{6 0 - 1 1 6}$ & $>\mathbf{1 1 6}$ \\
\hline $\begin{array}{c}\text { INSTRUMENTAL } \\
\text { INTENSITY }\end{array}$ & $\mathbf{I}$ & II-III & IV & $\mathbf{V}$ & VI & VII & VIII & IX & X+ \\
\hline
\end{tabular}

Figure 4. Close-up view of intensity distribution in the Kachchh region (see caption for Fig. 3).

Within $100 \mathrm{~km}$ of the fault, however, ground motions estimated from our MMI values are systematically higher than those predicted by the model, typically by 1-3 units. It is possible that most of this discrepancy is due to site response, which will tend to increase MMI on soil sites by at least 1 unit relative to that on rock sites. Other factors that may also be important are (1) the vulnerability of local buildings to shaking (2) a tendency for media accounts to focus on the most extreme damage in hard-hit regions, especially in large cities, and (3) the nature of the ground motions in an intraplate region. It is difficult to estimate the bias con- tributed by each effect. However, we consider it unlikely that moderate estimated MMI values (IV-VI) are significantly amplified because of building vulnerability, because these values reflect light damage (cracking of walls) and other effects (objects being knocked off shelves) that should not depend strongly on building type. It therefore appears likely that the other two factors account for more of the unit discrepancy, at least at close distances. Because news accounts generally focus on the most extreme rather than the typical damage in a region, it is not surprising that MMI values derived from media accounts will be systematically 

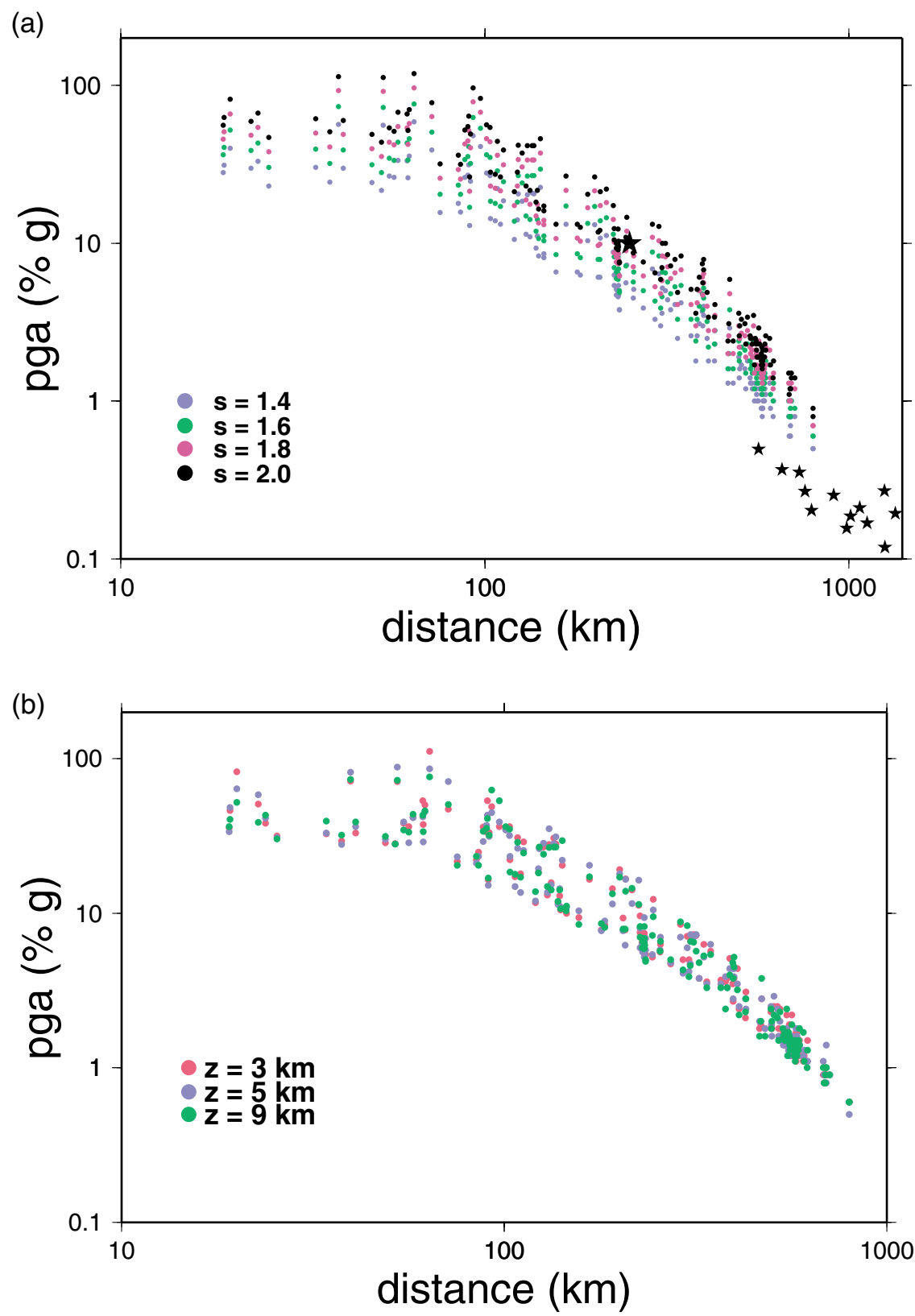

Figure 5. (a) PGA values predicted on rock by the finite-fault model of Beresnev and Atkinson (1997) for strength factors that vary between 1.4 and 2.0. (b) PGA values predicted on rock by the finite-fault model of Beresnev and Atkinson (1997) for models in which the rupture terminates at 3-, 5-, and 9-km depth.

higher than those determined from average effects, in the manner employed by the Wald et al. (1999) study. Even at low shaking levels, a media account might describe only the relately dramatic effects that occurred in a given location.

One must also consider the possibility that a PGA-MMI relationship determined for earthquakes in California is not appropriate for an intraplate region. In particular, it has been suggested that, by virtue of having a higher average stress drop, intraplate ground motions might be characterized by a higher level of high-frequency energy and therefore be more damaging (to some types of structures especially) than those from comparable earthquakes in interplate regions (e.g., Greig and Atkinson, 1993; Atkinson, 2001). To test this possibility, we recalculate predicted MMI values for a small number of locations by using relationships between MMI and response spectra determined by Atkinson and Sonley (2000). These relationships are also determined for earthquakes in California. However, Atkinson (2001) validates their applicability in intraplate regions by using the 1988 Saguenay earthquake and argues that the relationships are generally appropriate because frequency content is handled explicitly. Figure 8 presents the MMI results determined from both PGA 
(a)

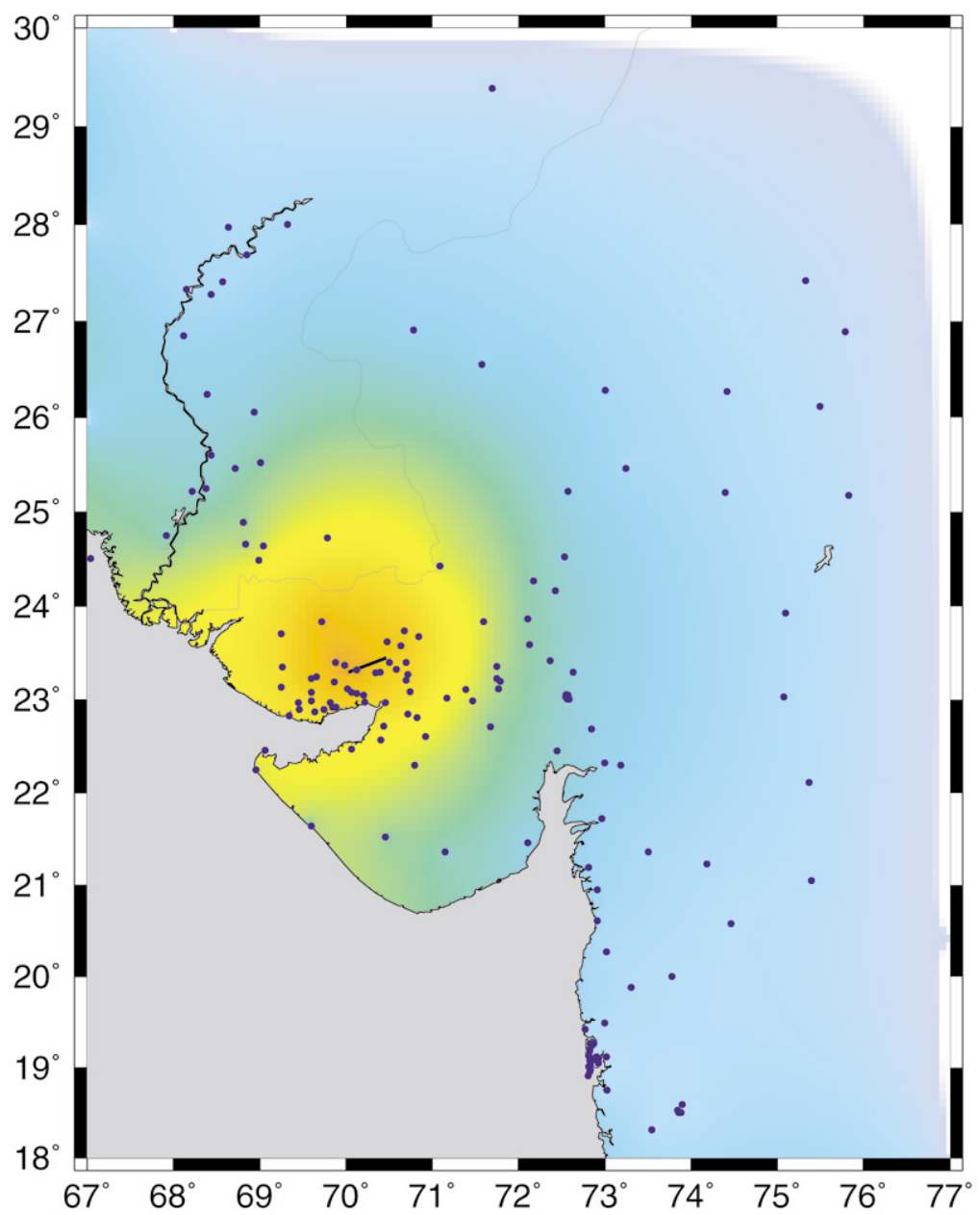

\begin{tabular}{|c|c|c|c|c|c|c|c|c|c|}
\hline PERCEIIED & Not felt & Weak & Light & Moderate & Strong & Very strong & Severe & Violent & Extreme \\
\hline $\begin{array}{c}\text { POTEKNTG } \\
\text { DAMAGE }\end{array}$ & none & none & none & Very light & Light & Moderate & Moderate/Heavy & Heavy & Very Heavy \\
\hline PEAK ACC.(\%g) & $<.17$ & $\mathbf{. 1 7 - 1 . 4}$ & $\mathbf{1 . 4 - 3 . 9}$ & $\mathbf{3 . 9 - 9 . 2}$ & $\mathbf{9 . 2 - 1 8}$ & $18-34$ & $34-65$ & $65-124$ & $>124$ \\
\hline PEAK VEL.(cm/s) & $<0.1$ & $0.1-1.1$ & $1.1-3.4$ & $3.4-8.1$ & $8.1-16$ & $\mathbf{1 6 - 3 1}$ & $\mathbf{3 1 - 6 0}$ & $\mathbf{6 0 - 1 1 6}$ & $>\mathbf{1 1 6}$ \\
\hline $\begin{array}{c}\text { INSTRUMENTAL } \\
\text { INTENSITY }\end{array}$ & $\mathrm{I}$ & II-III & IV & V & VI & VII & VIII & IX & X+ \\
\hline
\end{tabular}

Figure 6. Predicted ground motions on rock for models with strength factors of 1.6 (a) and 2.0 (b). Note that intensities on soil would be 1-2 units higher. (continued)

and response spectra, both on rock, and shows that the latter are indeed higher than the former. On average, the MMI values are increased by approximately 1 unit when the response spectra relations are used. If one considers the expected influence of site response, the MMIs predicted from response spectra are in reasonably good agreement with the observations.

\section{Implications for the 1811-1812 New Madrid Earthquakes}

The parallels between the Bhuj earthquake and the 1811-1812 New Madrid earthquakes are so striking as to have been commented on within days (or even hours) of the event. However, the extent to which the earthquakes and source regions are analagous has been the subject of some debate, as Gujarat is much closer to a plate boundary than is New Madrid. Both source regions appear to be failed rift systems that generate their largest earthquakes on thrust faults favorably oriented for slip in the current stress regime (e.g., Bendick et al., 2001). Also, both the Bhuj earthquake and the 7 February 1812, New Madrid earthquake appear to have been of similar size (Hough et al., 2000), with neither event generating an extensive surface rupture.

In its shaking effects, the Bhuj earthquake appears to have been very similar to the largest New Madrid event, 
(b)

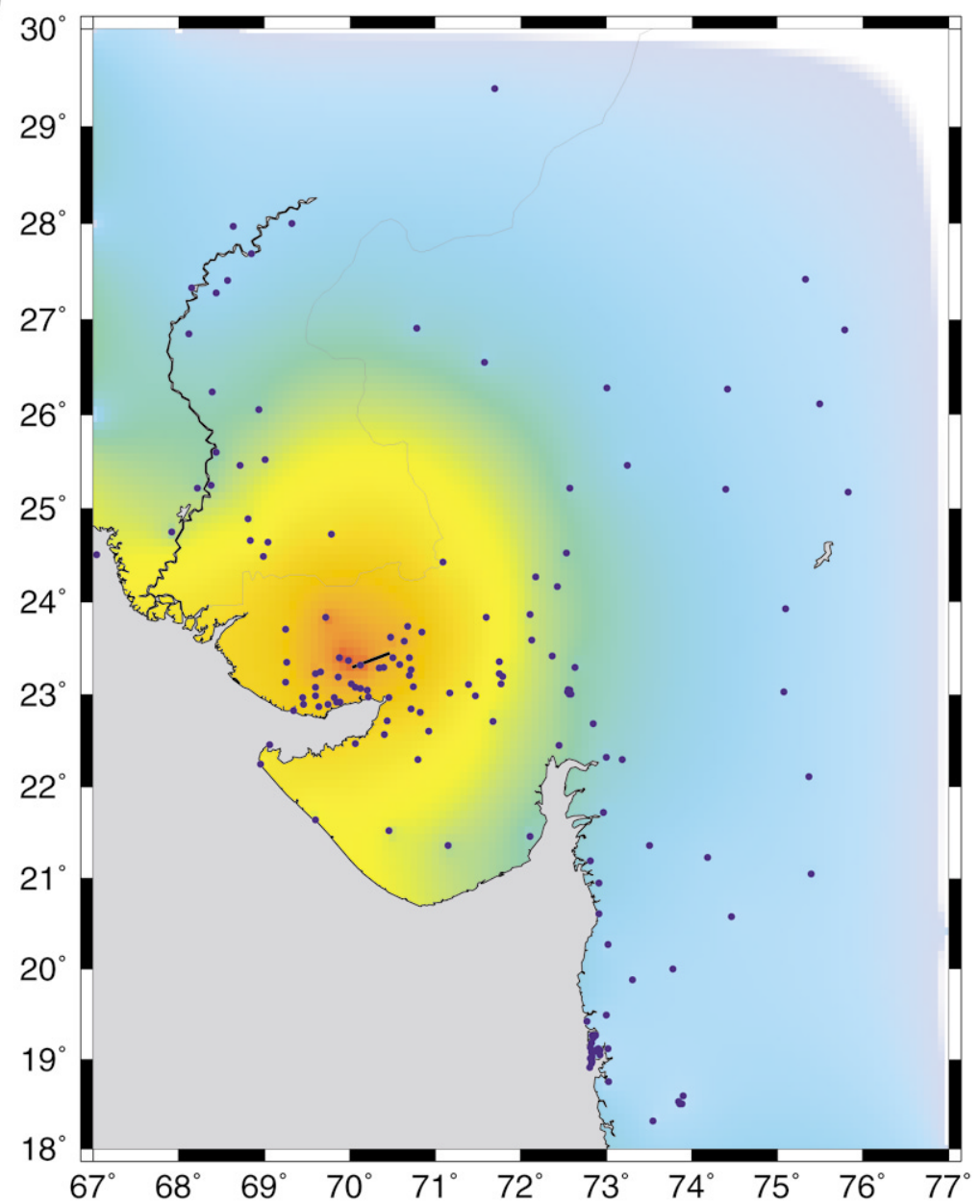

\begin{tabular}{|c|c|c|c|c|c|c|c|c|c|}
\hline $\begin{array}{l}\text { PERCEEVED } \\
\text { SHAKING }\end{array}$ & Not felt & Weak & Light & Moderate & Strong & Very strons & Severe & Violent & Extreme \\
\hline $\begin{array}{l}\text { POTENTIAL } \\
\text { DAMAEE }\end{array}$ & none & none & none & \begin{tabular}{|l|} 
Very light \\
\end{tabular} & Light & Moderate & Moderate/Heavy & Heavy & Very Heavy \\
\hline PEAK ACC. $(\% g)$ & $<.17$ & $.17-1.4$ & $1.4-3.9$ & $3.9-9.2$ & $9.2-18$ & $18-34$ & $34-65$ & $65-124$ & $>124$ \\
\hline PEAK & $<0.1$ & $0.1-1.1$ & $1.1-3.4$ & $3.4-8.1$ & $8.1-16$ & $16-31$ & $31-60$ & $60-116$ & $>116$ \\
\hline $\begin{array}{l}\text { INSTRUMNTAL } \\
\text { INTENSITY }\end{array}$ & 1 & II-III & IV & V & VI & VII & VIII & IX & $X_{+}$ \\
\hline
\end{tabular}

Figure 6. (Continued).

which, according to the interpretation of Hough et al. (2000), occurred on 7 February 1812. Hough et al. (2000) inferred an $M$ of 7.4-7.5 for this event; previous studies had inferred $M$ values as high as 8.0 (Johnston, 1996a, b).

The shaking effects of the Bhuj and the three principal 1811-1812 New Madrid mainshocks were very similar. All of these events were felt at coastal regions as far as $2000 \mathrm{~km}$ from the epicenter, all caused light damage at sediment sites as far as $600 \mathrm{~km}$ away, and all generated substantial liquefaction over an atypically large region. After the Bhuj earthquake, liquefaction was reported as far as $250 \mathrm{~km}$ from the fault, similar to the extent of liquefaction generated by the 1811-1812 New Madrid sequence (Tuttle et al., 2001a).

To compare the shaking effects of the Bhuj and New
Madrid earthquakes, we present a comparison of MMI data as a function of distance from this study and from Hough et al. (2000). Figure 9 presents the results for both the 16 December 1811 and the 7 February 1812, New Madrid events.

The comparisons illustrated in Figure 9 are complicated by the fact that the December and February New Madrid mainshocks occurred at approximately 2:15 a.m. and 3:45 a.m. (local time), whereas the Bhuj earthquake occurred later in the morning. Hough et al. (2000) assigned MMI values of IV for all locations at which these New Madrid events were reported as "felt," because intensity IV is the level of shaking at which a few people will be wakened. Hough et al. (2000) additionally find that the December event was not felt at approximately 15 locations at distances of $800-1900 \mathrm{~km}$. 


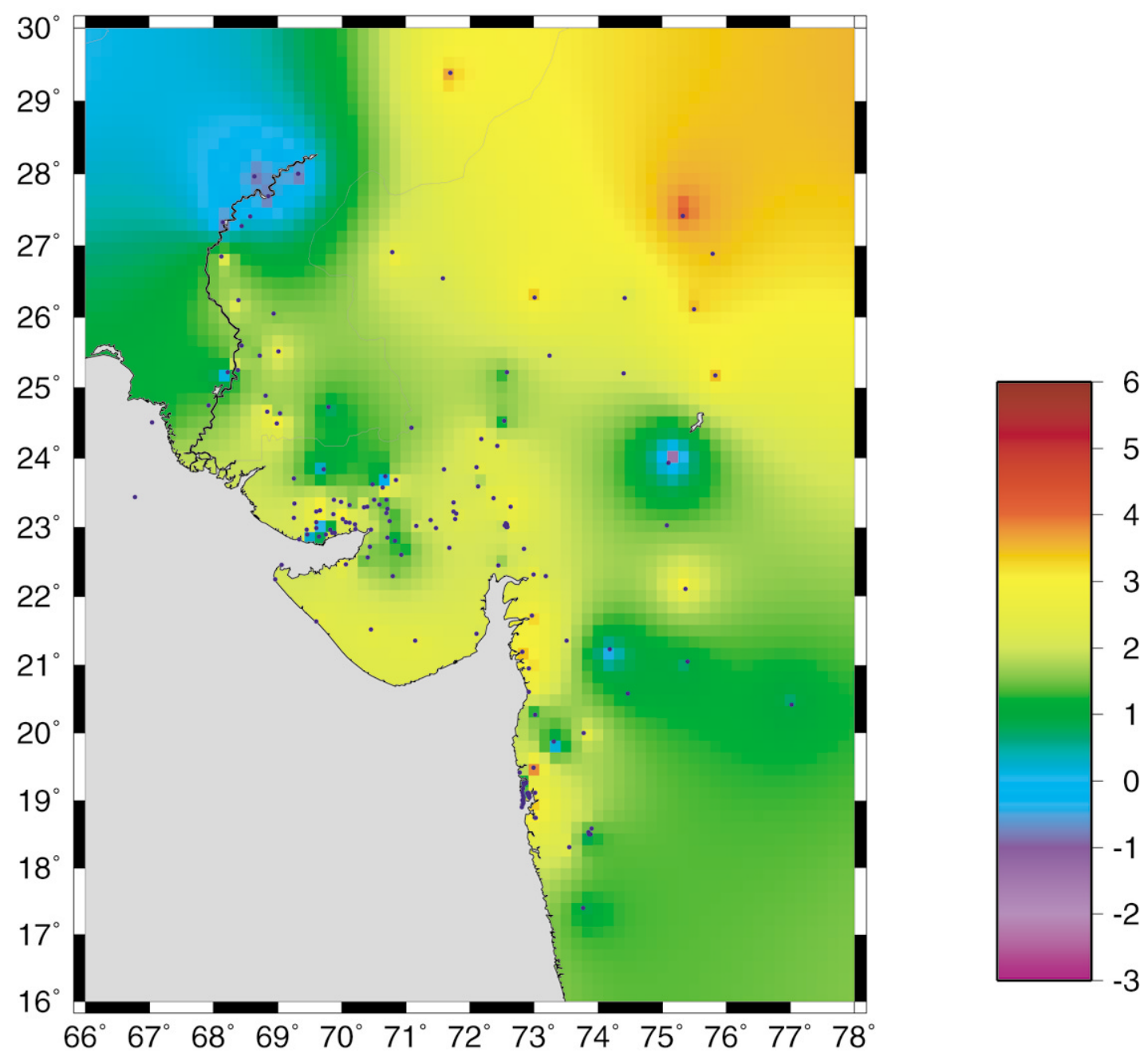

Figure 7. Residuals between our estimated MMI values for rock sites and those predicted by our preferred rupture model. For this figure, a different smoothing parameter $(0.5)$ is used. This value is chosen because it produces a smoother interpolation between isolated points where the residuals are constrained. (A smoothing value of 1.0 produces sharp local maxima and minima.)

(The February mainshock was felt to greater distances, but Hough et al. interpret some "not felt" reports for this event as well.) These observations are inferred to imply only an upper intensity bound of III.

The comparisons are further complicated by uncertainties regarding the exact fault planes of the New Madrid earthquakes. The distances shown in Figure 9 are calculated not to presumed fault planes, as is done for the Bhuj observations, but to crude estimates of the earthquakes' epicenters. The high MMI values for both New Madrid earthquakes at 50-100 km may therefore reflect the difference distance measurements used.

From Figure 9, we conclude that it is difficult to distinguish the Bhuj intensities from those from either of the New Madrid events, perhaps suggesting that the December and February New Madrid events were of comparable size to the Bhuj earthquake. However, the question of sampling biases remains. It appears that sediment-induced amplification caused pockets of high ground motions at regional distances during both the Bhuj and New Madrid events. However, because the population of the United States' mid-continent was heavily concentrated along rivers in the early 1800s, the low-intensity shaking field was not well sampled in this case. Intensity values may therefore be systematically higher than those of later earthquakes, including Bhuj, for which the population distribution is more even.

A comparison between New Madrid and Bhuj intensities is also valid only if the two regions are characterized by similar propagation characteristics. Although the Indian shield region and cratonic central/eastern North America were both considered stable continental regions in the seminal work of Johnston (1996a), Bakun (pers. comm. 2001) has shown that the intensities are generally higher for large earthquakes in eastern North America compared with comparable earthquakes in India. Indeed, the $Q(f)$ values used in this study imply lower values between 1 and $7 \mathrm{~Hz}$ than 


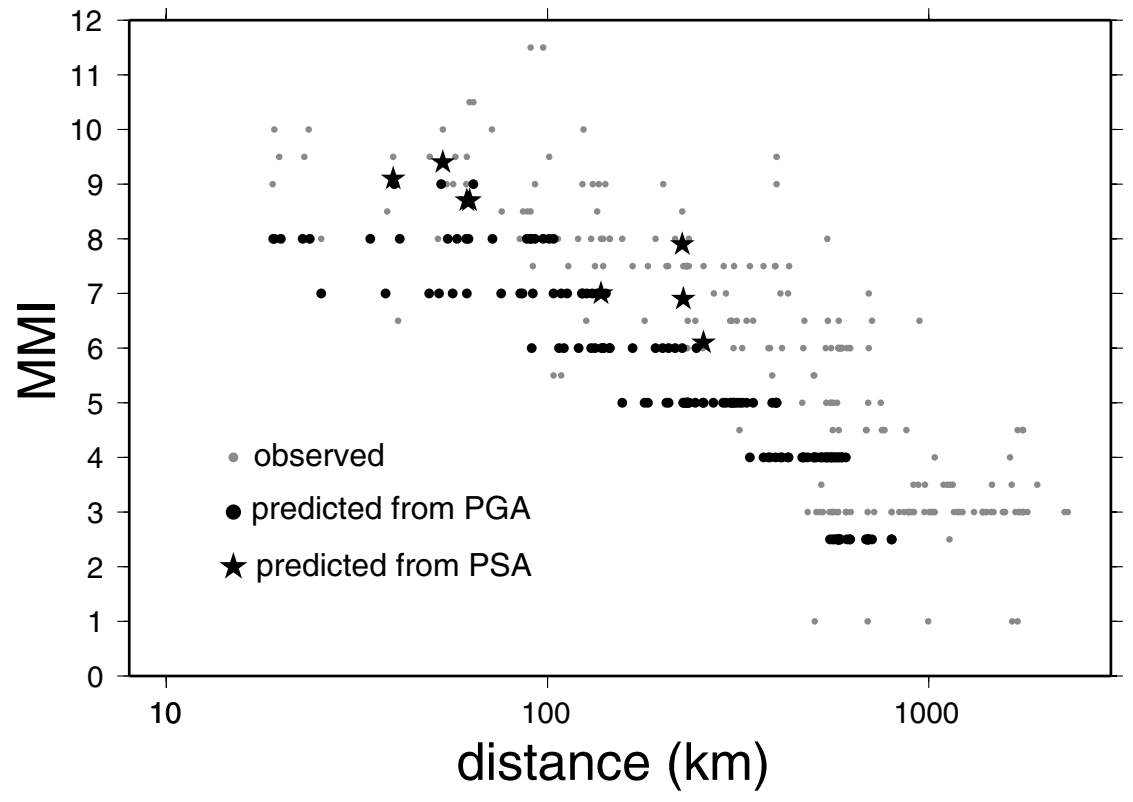

Figure 8. Our MMI values for the Bhuj earthquake are shown as a function of distance (small gray circles) along with predicted values calculated using an MMI-PGA relationship (large black circles) and one between MMI and response spectra (black stars).

$Q(f)$ determined by Benz et al. (1997) for both central and eastern North America (Fig. 10). Atkinson and Mereu (1992) also find higher $Q(f)$ values in southeastern Canada than those of Singh et al. (1999).

A relative calibration of MMI attenuation in India and North America is not yet available. We suggest, however, that the magnitude of the Bhuj earthquake, $M$ 7.6-7.7, represents a credible upper bound for the two largest New Madrid mainshocks, although we consider it entirely possible, considering sampling bias and attenuation issues, that the New Madrid events were somewhat smaller. When a more thorough comparison of intensity attenuation in India and North America is available, it should be possible to draw a more quantitative conclusion regarding the magnitude of the New Madrid mainshocks.

\section{Implications for the 1819 Allah Bund Earthquake}

The 1819 Allah Bund earthquake in the northern Rann of Kachchh was discussed at length by Oldham (1926) in one of his last important contributions. His interest in this event was initially stimulated by his efforts to complete his father's account of Indian earthquakes (Oldham, 1883) and by the discovery of Baker's profile (Baker, 1846) during a clean-out of the Bombay office of the Geographical Journal of Bombay in 1896. Baker's profile across the Allah Bund had been accidentally omitted by the editor from his narrative describing surface deformation but forms the basis of a subsequent surface-rupture parameter estimation by Bilham (1998).
Oldham collated newspaper reports of the 1819 event to produce an isoseismal contour map. This map was used by Richter (1958) to produce one of the first magnitude estimates for the event. His magnitude, 8.0, was derived from a comparison of the felt areas of the 1819 event with those of the 1905, 1934, and 1950 Indian earthquakes for which he had derived surface-wave magnitudes. Recent recalibrations of these magnitudes suggest that many are inflated (Chen and Molnar, 1983; Ambraseys and Bilham, 2000).

Attempts to quantify the magnitude of the 1819 event from Oldham's isoseismal data were subsequently attempted by Johnston and Kanter (1992) and by Bilham (1998). Magnitude estimates varied from 7.6 to 7.9. A geological estimate of the magnitude has been proposed by Rajendran and Rajendran (2001), based on the estimated rupture length and a surface-slip estimate of $3 \mathrm{~m}$. Bilham (1998) used Baker's profile to derive a geodetic moment magnitude of $7.7 \pm 0.2$.

The 2001 Bhuj earthquake stands to provide important new constraints on the magnitude of the 1819 event, in that the mechanisms and locations of the two events are very similar. In many cases, local construction practices have not changed. In some cases, the same historic structures were damaged by both events (e.g., the forts and town walls of Bhuj and Anjar). Yet there are important differences, in that some earthquake-resistant structures have been built in recent years; also, no concrete frame buildings existed in 1819.

A detailed intensity map for the 1819 earthquake is unavailable. However, Bilham (1998) does map sites that experienced severe and light damage, as well as sites at which the event was reportedly felt. We make crude MMI assign- 

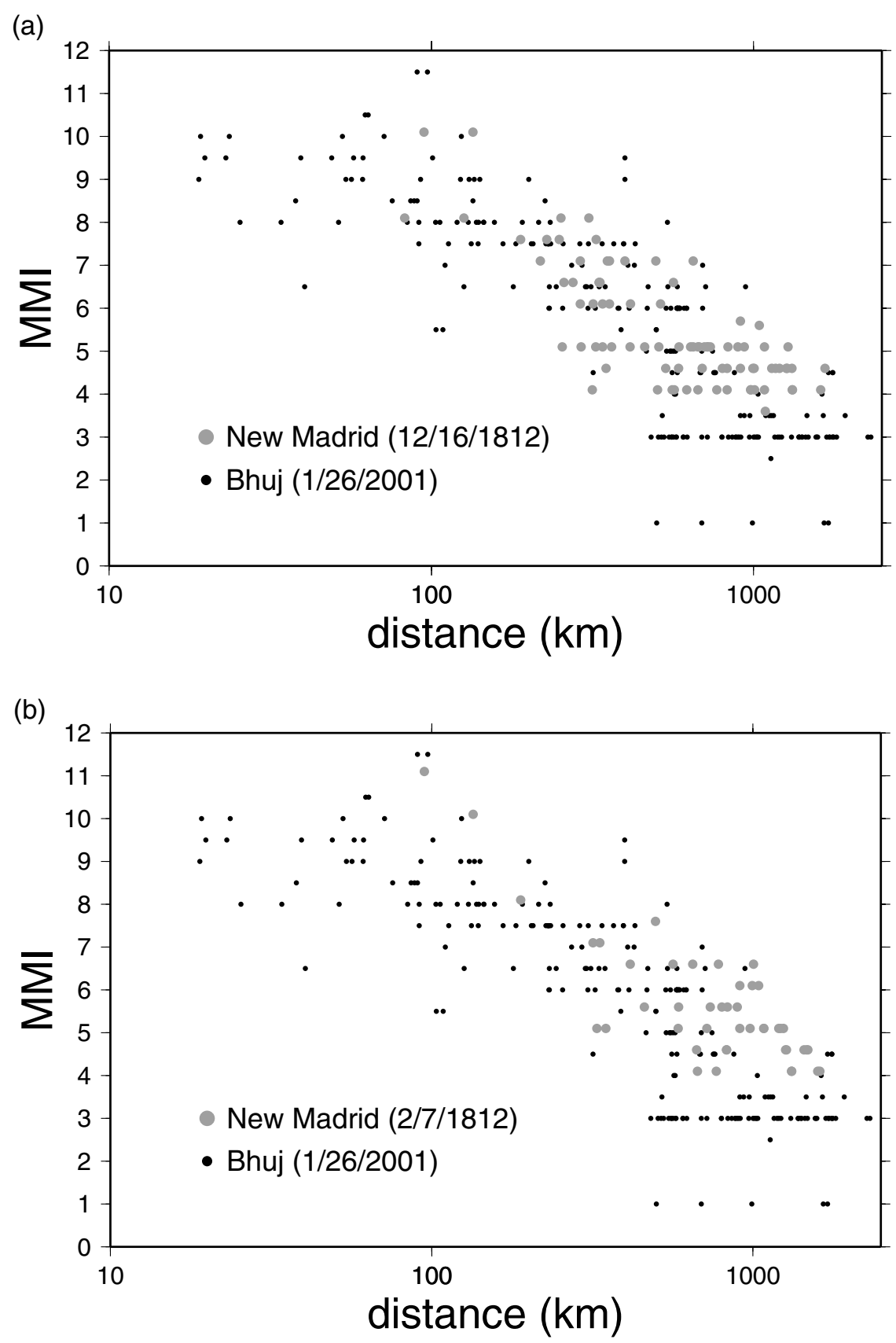

Figure 9. Our MMI values for the Bhuj earthquake are shown as a function of distance (small black circles) along with those determined by Hough et al. (2000) for the 16 December 1811 earthquake (large gray circles.) New Madrid MMI values are offset slightly upward for clarity.

ments of IX, VI, and III for these shaking levels, respectively (Fig. 11). A comparison of the isoseismal distribution of the 1819 and 2001 earthquakes shows that they are virtually indistinguishable in overall characteristics. Both events were felt lightly on the eastern coast of India; both caused light damage to distances of 500-600 km; and both caused heavy damage to distances of approximately $100 \mathrm{~km}$ (Fig. 11). (The extent of the high-intensity region is larger for the 1819 earthquake than it is for the Bhuj earthquake, but we attribute this to the sparsity of the 1819 data and our inability to assess precise MMI values for each site where "severe" damage occurred.)

We therefore conclude that the magnitude of the 1819 Allah Bund earthquake was also likely to have been very close to 7.6. This value is within the uncertainties of previous estimates but suggests that rupture dimensions and/or slip in 1819 may have been somewhat smaller than the values permitted by the higher geological and geodetic estimates. 


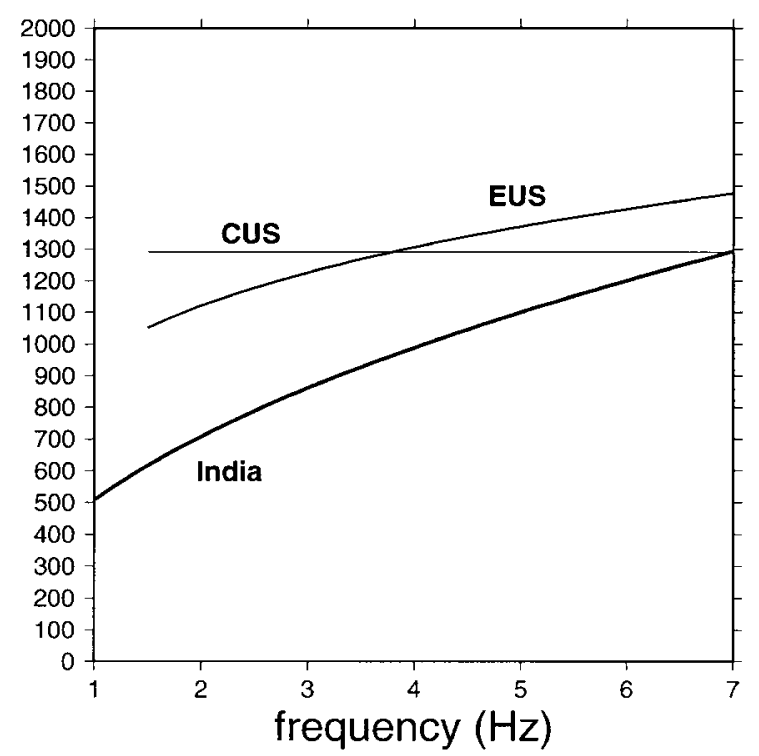

Figure 10. Attenuation function used in this study (Singh et al., 1999) is shown (dark line) along with $Q(f)$ inferred for the central United States (CUS) and eastern United States (EUS) by Benz et al. (1997).

\section{Discussion and Conclusions}

We have compiled media-based intensity maps for the 26 January 2001, Bhuj earthquake. These maps, based only on news accounts of the event, allow us to map the general distribution of shaking effects; they will also ultimately provide insight into the potential biases associated with determination of intensities based solely on media accounts. Such results are expected to be very useful, as the 2001 Bhuj earthquake has important implications for earthquake hazard, not only in India but also in other parts of the world where the source zones and/or the wave travel paths are similar (although the degree of similarity clearly bears further investigation). On the basis of our results and the similarity between their intensity distributions, we conclude that the 1819 Allah Bund earthquake had a magnitude very close to that of the 2001 Bhuj event: $7.6 \pm 0.1$. Our results also suggest that the magnitudes of the two largest 1811-1812 New Madrid earthquakes were slightly smaller than that of the Bhuj event, although the difference is difficult to quantify.

Our results show that, especially in the absence of modern instrumentation, MMI data can provide important information about the distribution of ground motions. As dis-

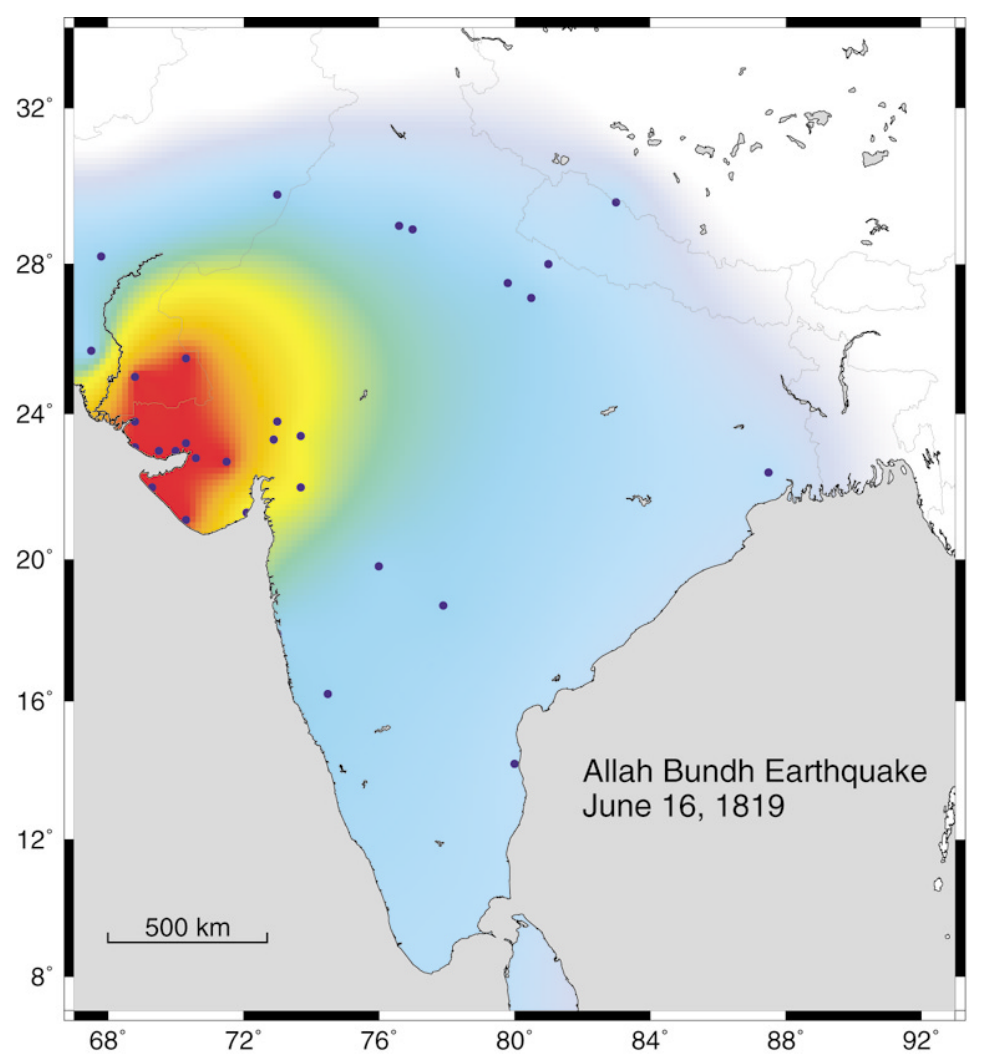

\begin{tabular}{|c|c|c|c|c|c|c|c|c|c|}
\hline PERCEIVED & Not felt & Weak & Light & Moderate & Strong & Very strong & Severe & Violent & Extreme \\
\hline SOAKENG & POIAL \\
DAMAGE & none & none & none & Very light & Light & Moderate & Moderate/Heavy & Heavy & Very Heavy \\
\hline PEAK ACC.(\%g) & $<.17$ & $.17-1.4$ & $1.4-3.9$ & $3.9-9.2$ & $9.2-18$ & $18-34$ & $34-65$ & $65-124$ & $>124$ \\
\hline PEAK VEL.(cm/s) & $<0.1$ & $0.1-1.1$ & $1.1-3.4$ & $3.4-8.1$ & $8.1-16$ & $16-31$ & $31-60$ & $60-116$ & $>116$ \\
\hline INSTRUMENTAL & I & II-III & IV & V & VI & VII & VIII & IX & X+ \\
\hline
\end{tabular}

Figure 11. Distribution of shaking effects from the 1819 Allah Bund earthquake, from Bilham (1999), compared with those determined in this study for the 2001 Bhuj earthquake. 
cussed earlier, site-response patterns are quite evident in the intensity distribution at both near and far distances. The overall felt distribution of the event also provides insights into the nature of $L g$-wave propagation. Hanks and Johnston (1992) showed that the far-reaching effects of central/eastern United States earthquakes can be explained by the efficient propagation of $L g$ waves (i.e., higher-mode surface waves) within cratonic North America. Kennett (1989) showed that $L g$ waves will propagate efficiently within a waveguide but will be disrupted when they encounter complexity such as crustal thickening. The felt area of the Bhuj earthquake is contained almost entirely within the Indian subcontinent. Our results therefore provide observational confirmation of the modeling results of Kennett (1989), that $L g$ waves are significantly disrupted by large-scale crustal complexity.

Our finite-fault-modeling results show that our estimated MMI values provide a good indication of the distribution of ground motions (PGA). Although the predicted hard-rock shaking level is lower than that inferred from macroseismic observations, we conclude that site response can explain most of the discrepancy. We have discussed three additional possible factors that might also contribute to the discrepancy: (1) extreme vulnerability of buildings in the Kachchh region, (2) a tendency of news accounts to focus on the most dramatic damage, and (3) the nature of the ground motions in intraplate crust. Although the first factor has been widely discussed, it is unlikely to account for the discrepancy in regions that experienced moderate (MMI IVVI shaking). We also note that the discrepancy is no larger in the epicentral region than at regional distances, which perhaps suggests that building vulnerability was not an important factor at close distances. This would not be an altogether surprising result, as building type and vulnerability are taken into account when MMI values are assigned.

At present, it is difficult to assess the effect of a possible media bias, although we consider to likely that such a bias did contribute to the discrepancy. A comparison with a survey-based intensity map will ultimately allow us to constrain the magnitude of this effect. This result will have implications for the interpretation of historical earthquakes for which the only available information is from printed media sources.

The final possibility, that the Bhuj ground motions were unusually damaging because of their high high-frequency energy, is interesting to consider. To compare predicted and estimate MMI values, we have used a relationship between MMI and PGA determined from recent large earthquakes in California. However, it has been suggested that large intraplate earthquakes might be more damaging than their interplate counterparts for reasons discussed earlier (e.g., Greig and Atkinson, 1993; Atkinson, 2001). We therefore also compared predicted and estimated MMI values by using a relationship between MMI and response spectral amplitudes (Atkinson and Sonley, 2000). Although also developed for California earthquakes, Atkinson (2001) concludes that the relationship is appropriate for earthquakes in eastern North
America, at least for distances of $150 \mathrm{~km}$ or less. Our results show that, using the response spectral regressions, our predicted ground motions imply rock MMI values approximately 1 unit higher than those estimated from the MMIPGA relationship. For soil sites, the predicted MMI values would be about 1 unit higher than for rock sites. Thus, there would be no significant discrepancy between observed and predicted MMI values.

Although much work remains to be done, the Bhuj earthquake provides important information to better understand the hazard posed by earthquakes that occur in and/or affect intercratonic regions. In addition to insights into the nature of source zones in low-strain-rate environments, the event provides invaluable new information with which ground motions from the past and future large intracratonic earthquakes can be better understood. The analysis presented here highlights the critical need to develop and test relationships between MMI and both PGA and response spectral ordinates for intraplate regions, and also to investigate in detail the attenuation of intensity in different intraplate regions.

\section{Acknowledgments}

We thank S. K. Singh, Kali Wallace, Martitia Tuttle, Tousson Toppozada, and Bill Bakun for helpful discussions and suggestions that greatly improved the manuscript; we also thank Igor Beresnev for helpful comments and for making available his ground-motion-modeling code. Maps were generated using GMT software [Wessel and Smith, 1991]. Research by S. Martin was supported by the Southern California Earthquake Center. SCEC is funded by NSF Cooperative Agreement EAR-8920136 and USGS Cooperative Agreements 14-08-0001-A0899 and 1434-HQ-97AG01718. The SCEC contribution number for this article is 608 .

\section{References}

Ambraseys, N., and R. Bilham (2000). A note on the Kangra $M_{\mathrm{s}}=7.8$ earthquake of 4 April 1905, Curr. Sci. 79, 101-106.

Atkinson, G. M. (2001). Linking historical intensity observations with ground motion relations for eastern North America, Seism. Res. Lett., 72, 560-574.

Atkinson, G. M., and R. F. Mereu (1992). The shape of ground motion attenuation curves in southeastern Canada, Bull. Seism. Soc. Am. 82, 2014-2031.

Atkinson, G., and E. Sonley (2000). Empirical relationships between modified Mercalli intensity and response spectra, Bull. Seism. Soc. Am. 90, 537-544.

Baker, W. E. (1846). Remarks on the Allah Bund and on the drainage of the eastern part of the Sind basin, Trans. Bombay Geogr. Soc. 7, 186188.

Bendick, R., R. Bilham, E. Fielding, V. Gaur, S. E. Hough, G. Kier, M. N. Kulkarni, S. Martin, K. Mueller, and M. Mukul (2001). The January 26, 2001 Bhuj, India earthquake, Seism. Res. Lett. 328-335.

Benz, H. M., A. Frankel, and D. M. Boore (1997). Regional $L g$ attenuation for the continental United States, Bull. Seism. Soc. Am. 87, 606-619.

Beresnev, I. A., and G. M. Atkinson (1997). Generic finite-fault model for ground-motion prediction in eastern North America, Bull. Seism. Soc. Am. 89, 608-625.

Beresnev, I. A., and G. M. Atkinson (2002). Source parameters of earthquakes in eastern and western North America based on finite-fault modeling, Bull. Seism. Soc. Am. 92, 695-710.

Bilham, R. (1999). Slip parameters for the Rann of Kachchh, India, 16 June 1819 , earthquake, quantified from contemporary accounts, in I. S. 
Stewart and C. Vita-Finzi (Editors), Coastal Tectonics, Geological Society London, 146, 295-318.

Bilham, R., and V. Gaur (2000). The geodetic contribution to Indian seismotectonics, Current Science 79, 1259-1269.

Chen, W. P., and P. Molnar (1983). Focal depths of intracontinental and intraplate earthquakes and their implications for the thermal and mechanical properties of the lithosphere, J. Geophys. Res. 88, 41834214.

Greig, G., and G. Atkinson (1993). Damage potential of eastern North American earthquakes, Seism. Res. Lett. 64, 119-137.

Hanks, T. C., and A. Johnston (1992). Common features of the excitation and propagation of strong ground motion for North American earthquakes, Bull. Seism. Soc. Am. 82, 1-23.

Hough, S. E., J. G. Armbruster, L. Seeber, and J. F. Hough (2000). On the modified Mercalli intensities and magnitudes of the 1811-1812 New Madrid, central United States earthquakes, J. Geophys. Res. 105, 23,869-23,864.

Horton, S., P. Bodin, A. Johnston, M. Withers, C. Chiu, A. Raphael, I. Rabak, Q. Maio, R. Smalley, J. Chiu, and C. Langston (2001). Source characteristics of aftershocks of the India Republic Day earthquake (abstract), EOS 82, 5256.

Johnston, A. C. (1996a). Seismic moment assessment of earthquakes in stable continental regions-II: historical seismicity, Geophys. J. Int. 125, 639-678.

Johnston, A. C. (1996b). Seismic moment assessment of earthquakes in stable continental regions-III: New Madrid 1811-1812, Charleston 1886, and Lisbon 1755, Geophys. J. Int. 126, 314-344.

Johnston, A. C., and L. R. Kanter (1992). Stable continental earthquakes, Sci. Am. 262, 68-75.

Kennett, B. L. N. (1989). $L g$-wave propagation in heterogeneous media, Bull. Seism. Soc. Am. 79, 860-872.

Mueller, K., and J. Pujol (2001). Three dimensional geometry of the Reelfoot blind thrust: implications for moment release and earthquake magnitude in the New Madrid seismic zone, Bull. Seism. Soc. Am. 91, 1563-1573.

Musson, R. M. W (1998). The Barrow-in-Furness earthquake of 15 February 1865: liquefaction from a very small magnitude event, Pure Appl. Geophys. 152, 733-745.

Oldham, T. (1883). Catalog of Indian earthquakes, Memoir Geol. Surv. India, 19, Geol. Surv. India, Calcutta, 163-215.

Oldham, R. D. (1926). The Cutch (Kachh) earthquake of 16th June, 1819 with a revision of the great earthquake of 12th June, 1897, Memoir Geol. Surv. India 46, 71-146.

Paul, J., R. Burgmann, V. K. Gaur, R. Bilham, K. M. Larson, M. B. Ananda, S. Jade, M. Mukal, T. S. Anupama, G. Satyal, and D. Kumar (2001). The motion and active deformation of India, Geophys. Res. Lett. 28, 647-651.

Rajendran, C. P., and K. Rajendran (2001). Characteristics of deformation and past seismicity associated with the 1819 Kutch earthquake, northwestern India, Bull. Seism. Soc. Am. 91, 407-426.
Richter, C. F. (1958). Elementary Seismology, W. H. Freeman, New York. Singh, S. K., M. Ordaz, R. S. Dattatrayam, and H. K. Gupta (1999). A spectral analysis of the 21 May 1997, Jabalpur, India, earthquake $\left(M_{\mathrm{w}}\right.$ 5.8) and estimation of ground motion from future earthquakes in the Indian shield region, Bull. Seism. Soc. Am. 89, 1620-1630.

Stover, C. W., and J. L. Coffman (1993). Seismicity of the United States, 1568-1989 (revised), U.S. Geol. Surv. Prof. Pap., 1527.

Tuttle, M. P., J. V. Hengesh, W. R. Lettis, K. B. Tucker, S. L. Deaton, and J. D. Frost (2002). Observations and comparisons of liquefaction features and related effects induced by the Bhuj Earthquake, Earthquake Spectra (in press).

Tuttle, M. P., A. Johnston, G. Patterson, K. Tucker, C. P. Rajendran, K. Rajendran, M. Thakkar, and E. Schweig (2001b). Liquefaction induced by the 2001 Republic Day Earthquake, India, Seism. Res. Lett. 72, 397.

Wald, D. J., V. Quitoriano, T. H. Heaton, and H. Kanamori (1999). Relationships between peak ground acceleration, peak ground velocity, and modified Mercalli intensity in California, Earthquake Spectra 15, 557-564.

Wessel, P., and W. H. F. Smith (1991). Free software helps map and display data, EOS 72, 441, 445.

Yagi, Y., and M. Kikuchi (2001). Results of rupture process for January 26, 2001, western India earthquake ( $M_{\mathrm{s}}$ 7.9), http://wwweic.eri. u-tokyo.ac.jp/yuji/southindia/index.html (revised 9 March 2001; last accessed 1 June 2001).

U.S. Geological Survey

525 S. Wilson Ave.

Pasadena, California 91106

(S.E.H.)

Fergusson College

Pune 411004

Maharashtra, India

(S.M.)

Department of Geoogical Sciences

2200 Colorado Avenue

University of Colorado

Boulder, Colorado 80309-0399

(R.B.)

Carleton University

Department of Earth Sciences

Ottawa, Canada

(G.M.A.)

Manuscript received 12 October 2001 